\title{
COVID-19 dynamics considering the influence of hospital infrastructure: an investigation into Brazilian scenarios
}

\author{
Pedro M. C. L. Pacheco • Marcelo A. Savi • Pedro V. Savi
}

Received: 5 December 2020 / Accepted: 22 February 2021 / Published online: 13 March 2021

(C) The Author(s), under exclusive licence to Springer Nature B.V. part of Springer Nature 2021

\begin{abstract}
COVID-19 dynamics is one of the most relevant subjects nowadays, and, in this regard, mathematical modeling and numerical simulations are of special interest. This paper describes COVID-19 dynamics based on a novel version of the susceptibleexposed-infectious-removed model. Removed population is split into recovered and death populations allowing a better comprehension of real situations. Besides, the total population is reduced based on the number of deaths. Hospital infrastructure is also included into the mathematical description allowing the consideration of collapse scenarios. Initially, a model verification is carried out calibrating system parameters with data from China outbreak that is considered a benchmark due the availability of data for the entire cycle. Afterward, Brazil outbreak is of concern, calibrating the model and developing
\end{abstract}

\section{P. M. C. L. Pacheco}

Department of Mechanical Engineering, Centro Federal de Educação Tecnológica Celso Suckow da Fonseca CEFET/RJ, Rio de Janeiro 20.271.110, Brazil e-mail: pedro.pacheco@cefet-rj.br

M. A. Savi $(\varangle) \cdot$ P. V. Savi

Center for Nonlinear Mechanics, COPPE - Department of Mechanical Engineering, Universidade Federal do Rio de Janeiro, P.O. Box 68.503, Rio de Janeiro,

RJ 21.941.972, Brazil

e-mail: savi@mecanica.coppe.ufrj.br

P. V. Savi

e-mail: pedrov.savi@mecanica.coppe.ufrj.br numerical simulations. Results show several scenarios highlighting the importance of social isolation and hospital infrastructure. System dynamics has a strong sensitivity to transmission rate showing the importance of numerical simulations to guide public health decision strategies. Results also show that complex dynamical responses can emerge due to the oscillations of the transmission rate, being associated with distinct infection subsequent waves.

Keywords COVID-19 - Coronavirus · NCoV . Nonlinear dynamics · Mathematical models . Population dynamics

\section{Introduction}

Coronavirus disease 2019 (COVID-19) was discovered in 2019, becoming a pandemic that is promoting a dramatic reaction all over the world. Several uncertainties are associated with all the aspects of this disease including clinical evolution and contamination processes. Nonlinear dynamics of biological and biomedical systems is the objective of several researches that can be based either on mathematical modeling or on time series analysis [22].

Mathematical modeling is an interesting approach that can allow the evaluation of different scenarios, furnishing information for a proper support for health 
system decisions. In particular, coronavirus spread can be described by mathematical models that allow for the nonlinear dynamics analysis, representing different populations related to the phenomenon $[6,7,12]$.

In principle, reality can be considered indescribable, and therefore, it cannot be represented by mathematical models. Nevertheless, mathematical models can be understood as a reality caricature that can be useful to achieve specific goals and therefore being able to describe reality. In this regard, it can help for either qualitative understanding, establishing dynamical patterns, or quantitative predictions. Based on this idea, mathematical modeling is an art in the Picasso's sense, expressed by his remarkable sentence: "art is a lie that makes us realize truth."

Different kinds of models can be employed for the COVID-19 dynamics. Rihan et al. [19] described the dynamics of coronavirus infection in human, establishing interaction among human cells and the virus. Chen et al. [8] developed a mathematical model for calculating the transmissibility of the virus considering a simplified version of the bats-hosts-reservoirpeople transmission model, defined as a reservoirpeople model. Li et al. [14] estimated characteristics of the epidemiologic time distribution, exploiting some pattern trends of transmission propagation. Riou and Althaus [20] exploited the pattern of human-tohuman transmission of novel coronavirus in Wuhan, China. Two key parameters are considered: basic reproduction number that defines the infectious propagation, and the individual variation in the number of secondary cases. Uncertainty quantification tools were employed to define the transmission patterns. Zuo et al. [26] proposed a statistical model comparing the COVID-19 dynamics in several Asian countries. Car et al. [5] investigated COVID-19 dynamics from time series, building a dataset for training a multilayer perceptron artificial neural network and showing that artificial intelligence could have a good agreement with real data.

Susceptible-exposed-infectious-removed (SEIR) models are an interesting approach to deal with the mathematical modeling of infectious diseases, especially coronavirus transmission. Wu et al. [25] investigated Wuhan, China case, evaluating domestic and international spread outbreak. Lin et al. [15] proposed a model considering individual reaction, governmental action and emigration. The model is based on the original work of $\mathrm{He}$ et al. [10] that proposed a model to describe the 1918 influenza. Savi et al. [21] investigated the SEIR model applied to different countries, exploiting some aspects of Brazilian scenarios.

COVID-19 scenarios all over the world are becoming dramatic due to the absence of effective drugs and/ or vaccines. Even with vaccines, there are difficulties due to the distribution and their efficacy. Since it is possible that this general scenario is persisting for an unknown period of time or new coronavirus variants appears, it is required that governments need to implement alternative strategies, known as non-pharmaceutical interventions (NPIs), to contain the spread of coronavirus infection in the population. These strategies include governmental interventions related to the close of education system, induce social isolation and voluntary quarantine. This suggests the inclusion of other variables on the mathematical modeling in order to have better adjustments with real data and to furnish useful information for decision making.

In this regard, hospital infrastructure and the number of deaths seem to be essential points to be included on mathematical modeling. The literature presents some research efforts related to the dynamics of COVID-19 pandemic progress considering different scenarios of the NPIs for reducing transmission of the virus, as well as the hospital infrastructure necessary to take care of the infectious population $[4,9,15,16,18,24]$.

This contribution proposes a novel mathematical model to describe the general propagation of the coronavirus. The idea is to use the SEIR framework including different novel aspects: Removed population is represented by two populations-recovered and deaths; description of hospital infrastructure; and total population varies according to the number of deaths. Based on this model, numerical simulations are carried out. Initially, a model verification is carried out considering infected population evolution of China, considered as a benchmark case due to the availability of data for the entire cycle. Afterward, Brazilian data are employed to calibrate the model and the COVID19 nonlinear dynamics is investigated treating different scenarios. Results show that complex responses can emerge due to oscillations on the transmission rate, defining infection subsequent waves. 


\section{Mathematical model}

A frame-by-frame description of the COVID-19 dynamics can be represented by a set of differential equations of the form $\dot{x}=f(x), x \in \mathfrak{R}^{n}$, where $x \in \mathfrak{R}^{n}$ represents a set of state variables that describe the phenomenon. The description of COVID-19 dynamics defines its propagation considering different kinds of populations. An interesting alternative for this aim is the susceptible-exposed-infectious-removed (SEIR) framework model. The populations are defined considering that $S$ is the susceptible population, $E$ is the exposed population, $I$ is the infectious population, and two removed populations: recovered, $R_{\mathrm{C}}$, and death, $R_{\mathrm{D}}$. Under this assumption, the total population is $N=S+E+I+R_{\mathrm{C}}+R_{\mathrm{D}}$. Besides, the total population contains two classes: $D$ is a public perception of risk regarding severe cases and deaths; and $C$ represents the number of reported and non-reported cases. Another important observation is that population is reduced due to deaths, and therefore, $N$ is reduced based on the increase in death population, $R_{\mathrm{D}}$, with a rate, $\dot{R}_{\mathrm{D}}$.

Based on this, it is possible to establish a conceptual model of the COVID-19 dynamics presented in Fig. 1, which allows one to write the following governing equations:

$\dot{S}=-\beta \frac{S I}{N}$

$\dot{E}=\beta \frac{S I}{N}-\sigma E$

$\dot{I}=\sigma E-\left(\gamma_{\mathrm{C}}+\gamma_{\mathrm{D}}\right)\left(I-I^{\mathrm{H}}\right)-\gamma_{\mathrm{D}}^{\mathrm{H}} I^{\mathrm{H}}$

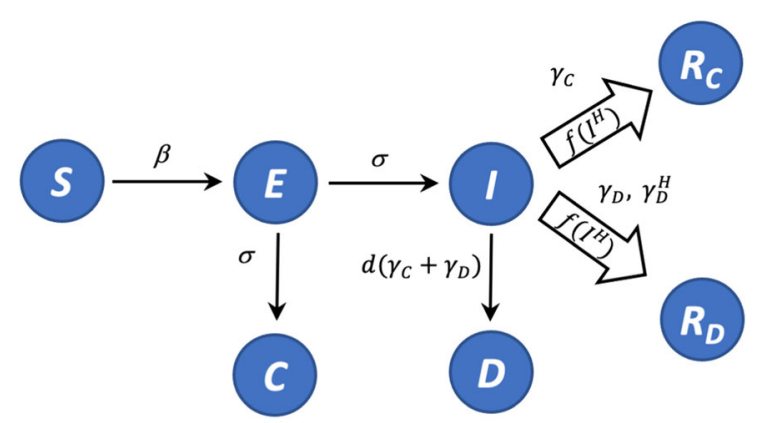

Fig. 1 Conceptual model for COVID-19 dynamics based on SEIR framework

$$
\begin{aligned}
& \dot{R}_{\mathrm{C}}=\gamma_{\mathrm{C}}\left(I-I^{\mathrm{H}}\right) \\
& \dot{R}_{\mathrm{D}}=\gamma_{\mathrm{D}}\left(I-I^{\mathrm{H}}\right)+\gamma_{\mathrm{D}}^{\mathrm{H}} I^{\mathrm{H}} \\
& \dot{D}=d\left(\gamma_{\mathrm{C}}+\gamma_{\mathrm{D}}\right) I-\lambda D \\
& \dot{C}=\sigma E \\
& \dot{N}=-\dot{R}_{\mathrm{D}} .
\end{aligned}
$$

It should be pointed out that COVID-19 dynamics has spatiotemporal characteristics that are not treated by this set of governing equations. Hence, the proposed model represents a kind of average behavior that needs a proper adjustment to match real data. A more realistic analysis would consider this model as a unit cell of a network with interaction among the cells. But this average behavior can be useful for different goals.

Another point that should be highlighted is that the model considers the effect of available hospital infrastructure to take care of the infectious population. Therefore, it is defined a subpopulation of the infectious, $I^{\mathrm{H}}$, which represents the part of the infectious that needs hospital assistance but does not have access due to the lack of infrastructure. This is described by function $\langle\cdot\rangle$ in order to represent the number of unavailable hospital assistance for the infectious population:

$I^{\mathrm{H}}=\left\langle\rho I-\widehat{N}_{\mathrm{H}}\right\rangle= \begin{cases}\rho I-\widehat{N}_{\mathrm{H}}, & \text { if } \rho I>\widehat{N}_{\mathrm{H}} \\ 0, & \text { if } \rho I \leq \widehat{N}_{\mathrm{H}}\end{cases}$

where $\rho$ is the percentage of the population inside the group that needs hospital assistance and $\widehat{N}_{\mathrm{H}}$ represents the number of available hospital infrastructure described as a function of time with the aid of a step function as follows and as shown in Fig. 2:

$$
\begin{aligned}
\widehat{N}_{\mathrm{H}} & =N_{\mathrm{H}}^{(i)} H\left(t-T_{\mathrm{H}}^{(i)}\right) \\
& =\left\{\begin{array}{cc}
N_{\mathrm{H}}^{(1)}, & \text { if } t \leq T_{\mathrm{H}}^{(1)} \\
N_{\mathrm{H}}^{(2)}, & \text { if } T_{\mathrm{H}}^{(1)}<t \leq T_{\mathrm{H}}^{(2)} \\
N_{\mathrm{H}}^{(3)}, & \text { if } T_{\mathrm{H}}^{(2)}<t \leq T_{\mathrm{H}}^{(3)} \\
\vdots &
\end{array}\right.
\end{aligned}
$$

where $N_{\mathrm{H}}^{(i)}$ represents the number of available hospital infrastructure until the time instant $T_{\mathrm{H}}^{(i)}$. Under this 


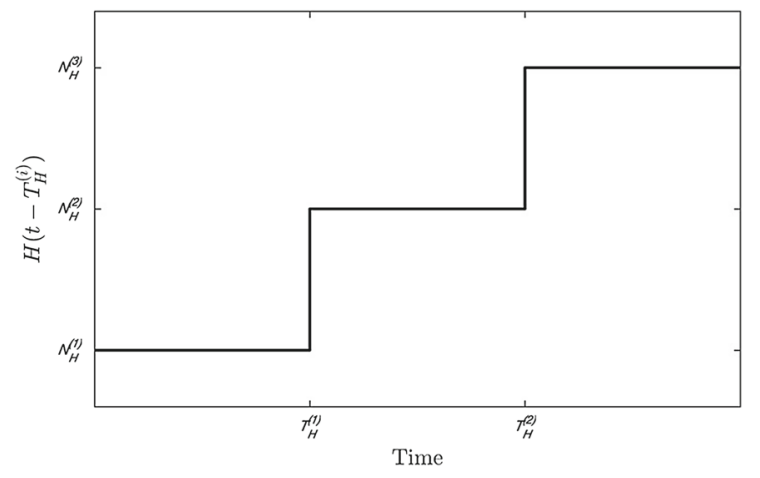

Fig. 2 Step function employed to consider parameter variations through time

assumption, time instant $T_{\mathrm{H}}^{(i)}$ represents the moment when the infrastructure is changed due to some action.

The transmission rate is represented by function of time, $\beta=\beta(t)$, which considers social isolation induced by governmental action, represented by $(1-\alpha)$, and the individual action, represented by the function $\delta$. Therefore, the transmission rate is modeled as follows: $\beta=\beta(t)=\widehat{\beta}_{0}(1-\widehat{\alpha}) \delta$

where $\widehat{\beta}_{0}=\beta_{0}^{(i)} H\left(t-T_{\beta_{0}}^{(i)}\right)$ represents the nominal transmission rate that is also a function of time, being described with the aid of a step function $H\left(t-T_{\beta_{0}}^{(i)}\right)$, similar to the one previously defined. The same strategy is employed to define social isolation induced by governmental actions, with the lockdown as the extreme case. Therefore, the following expression is written:

$\widehat{\alpha}=\alpha_{i} H\left(t-T_{\text {Gov }}^{(i)}\right)$

where different steps are considered defined by time instants $T_{\mathrm{Gov}}^{(i)}$.

In addition, individual action is represented by

$\delta=\left(1-\frac{D}{N}\right)^{\kappa}$

in which the intensity of responses is defined by parameter $\kappa$. It should be pointed out that the different values of transmission rate are closely related to the

Table 1 Model parameters for the simulations

\begin{tabular}{llc}
\hline Parameter & Description & Value \\
\hline$\gamma_{\mathrm{D}}$ & Death rate & $\gamma_{\mathrm{D}}=\left(\frac{C_{\mathrm{D}} / C_{I}}{1-R_{\mathrm{D}} / C}\right) \gamma_{\mathrm{C}}$ \\
$\gamma_{\mathrm{D}}^{\mathrm{H}}$ & Hospital assistance rate & 0.5 \\
$\rho$ & Percentage of the population inside the group that needs hospital assistance & $0.15(15 \%)$ \\
$d$ & Perception of severe cases and deaths & 0.2 \\
$\lambda^{-1}$ & Mean duration of public reaction & 11.2 days \\
$\kappa$ & Transmission rate parameter associated with individual actions & 1117.3 \\
\hline
\end{tabular}

Table 2 Model parameters for China

\begin{tabular}{llc}
\hline Parameter & Description & Value \\
\hline$\beta_{0}$ & Nominal transmission rate & 0.514 \\
$\sigma^{-1}$ & Mean latent period & 3 days \\
$\gamma_{\mathrm{C}}^{-1}$ & Mean recovered period & 5 days \\
$\gamma_{\mathrm{D}}$ & Death rate & $4.082 \times 10^{-3}$ \\
$\alpha_{i}$ & Transmission rate parameter associated with social isolation induced by governmental actions & {$[0,0.4239,0.8478]$} \\
$T_{\text {Gov }}^{(i)}$ & Transmission rate time parameter associated with social isolation induced by governmental actions & {$[0,13,20]$ days } \\
\hline
\end{tabular}




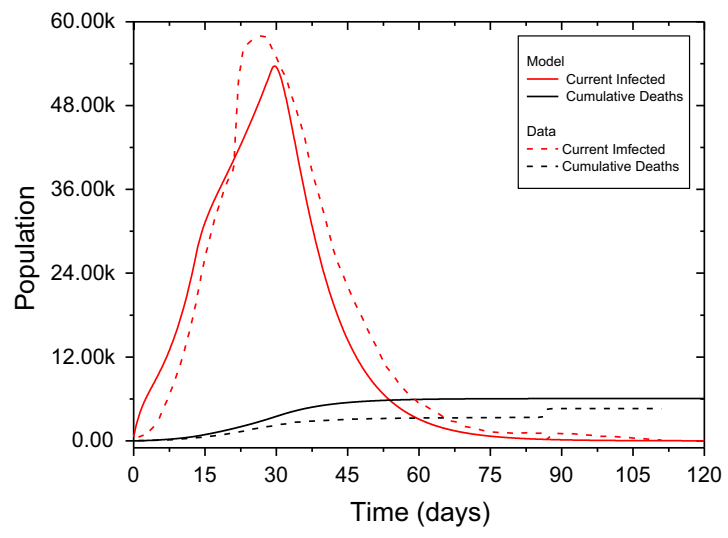

(a)

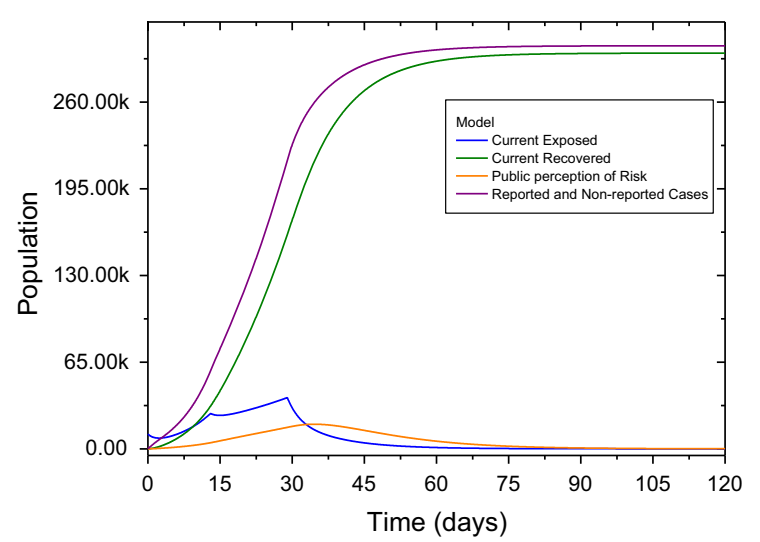

(b)

Fig. 3 Model verification based on China actual data: a comparison between numerical results and data for infected and cumulative death population through time and $\mathbf{b}$ other model variables evolution

social isolation. All these parameters need to be adjusted for each place, being essential for the COVID-19 description.

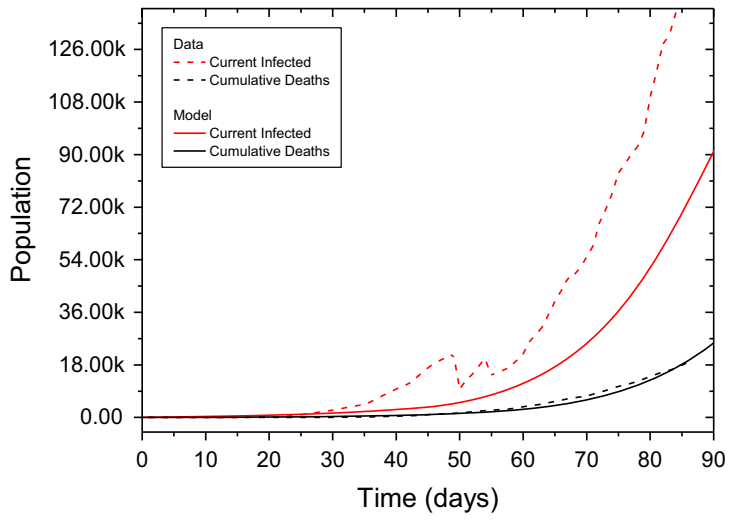

(a)

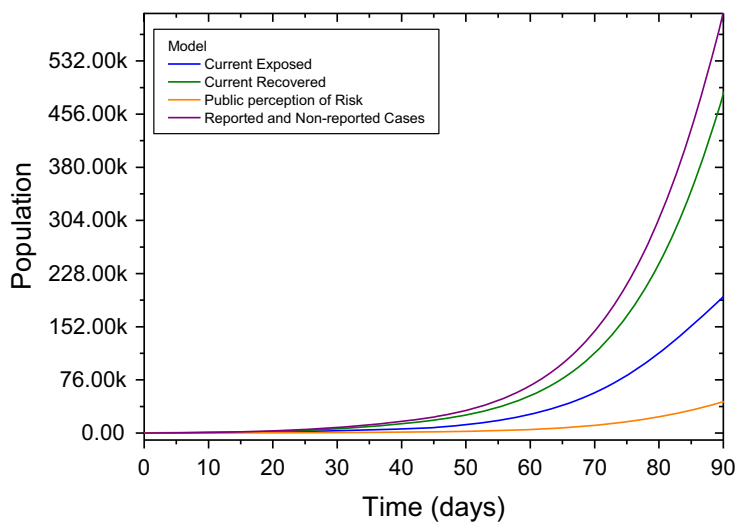

(b)

Fig. 4 Model verification based on Brazil actual data: a comparison between numerical results and data for infected and cumulative death population through time and $\mathbf{b}$ other model variables evolution

The following parameters are considered on the governing equations: $\sigma$ is the mean latent period; $d$ is the perception of severe cases and deaths; $\lambda$ is the mean duration of public reaction. Three parameters are adopted in order to describe the removed

Table 3 Model parameters for Brazil

\begin{tabular}{llc}
\hline Parameter & Description & Value \\
\hline$\beta_{0}$ & Nominal transmission rate & 1.020 \\
$\sigma^{-1}$ & Mean latent period & 5.2 days \\
$\gamma_{\mathrm{C}}^{-1}$ & Mean recovered period & 2.9 days \\
$\gamma_{\mathrm{D}}$ & Death rate & $1.815 \times 10^{-2}$ \\
$\alpha_{i}$ & Transmission rate parameter associated with social isolation induced by governmental actions & {$[0,0.40,0.30]$} \\
$T_{\text {Gov }}^{(i)}$ & Transmission rate time parameter associated with social isolation induced by governmental actions & {$[0,15,45]$ days } \\
\hline
\end{tabular}




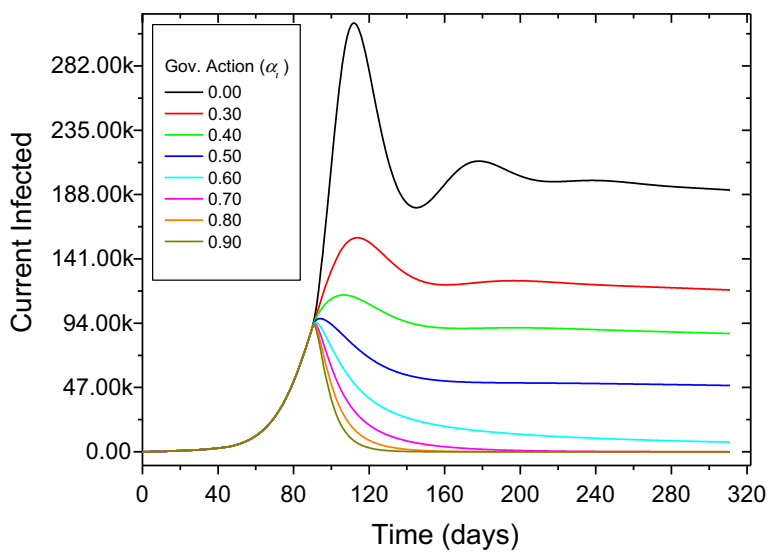

(a)

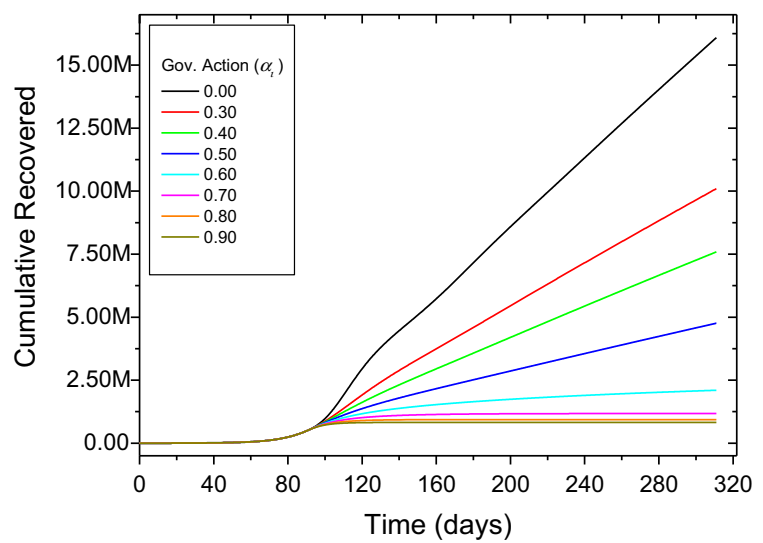

(c)

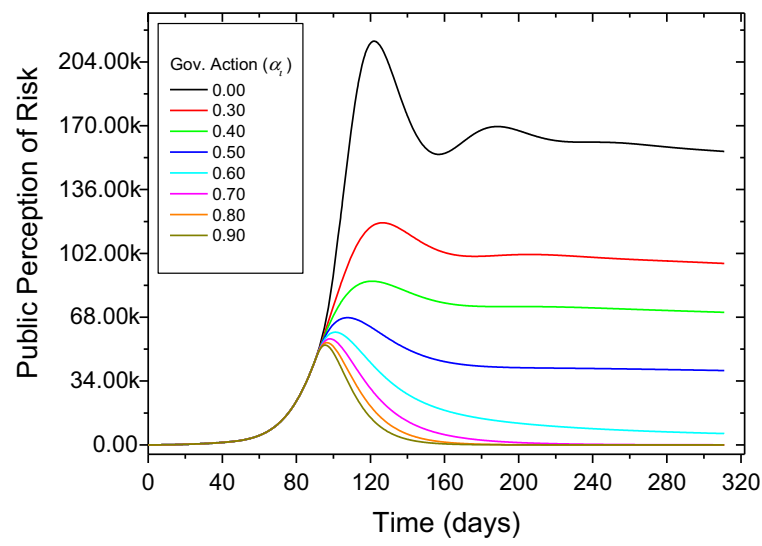

(e)

Fig. 5 Population dynamics considering different social isolation induced by governmental actions: a current exposed, b current infected, $\mathbf{c}$ cumulative recovered, $\mathbf{d}$ cumulative deaths,

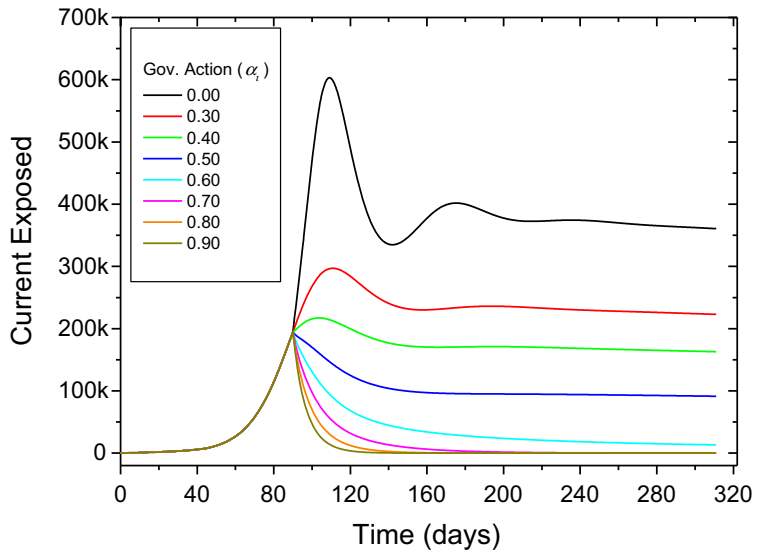

(b)

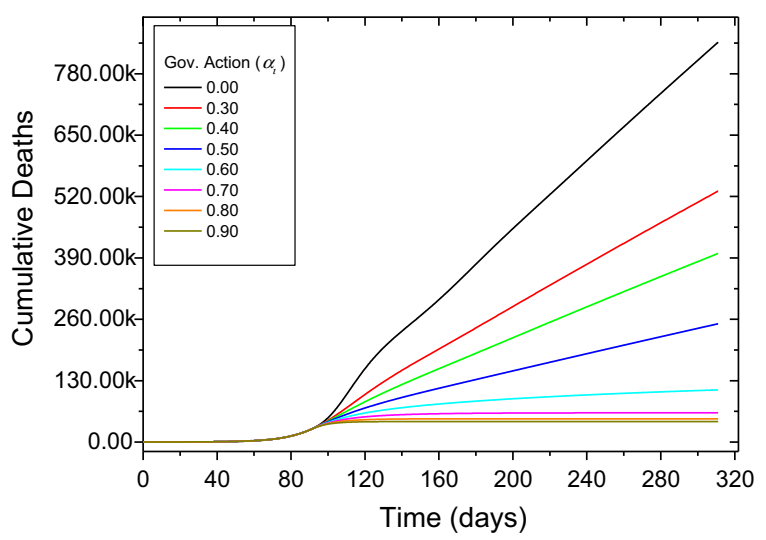

(d)

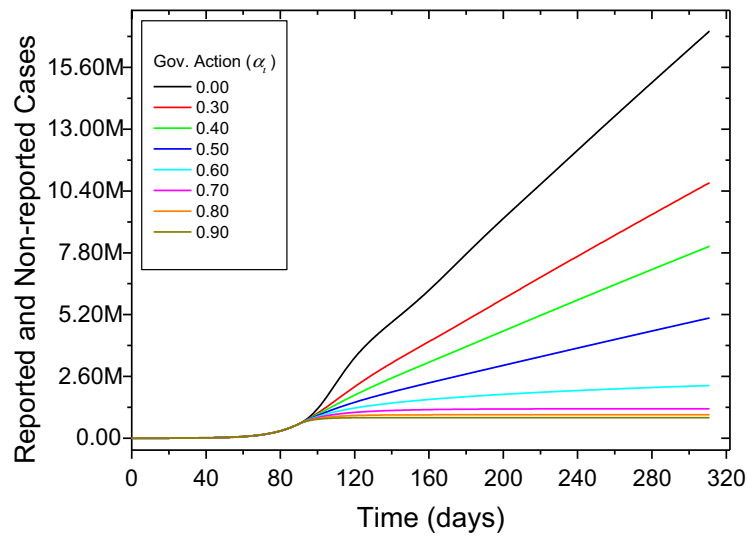

(f)

e public perception risk and $\mathbf{f}$ reported and non-reported cases. Hospital infrastructure without any restriction 


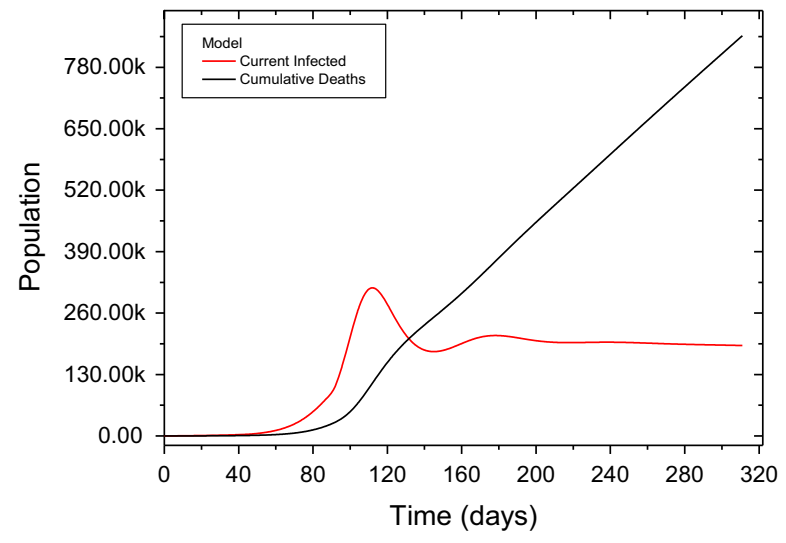

(a)

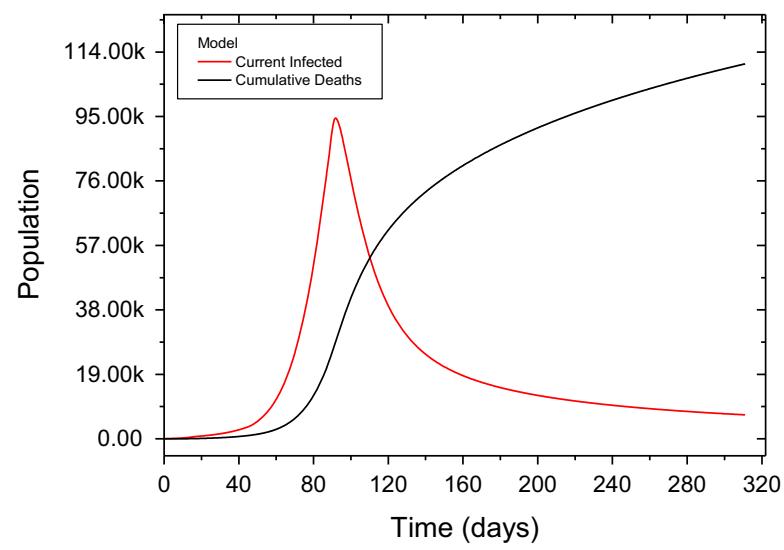

(c)

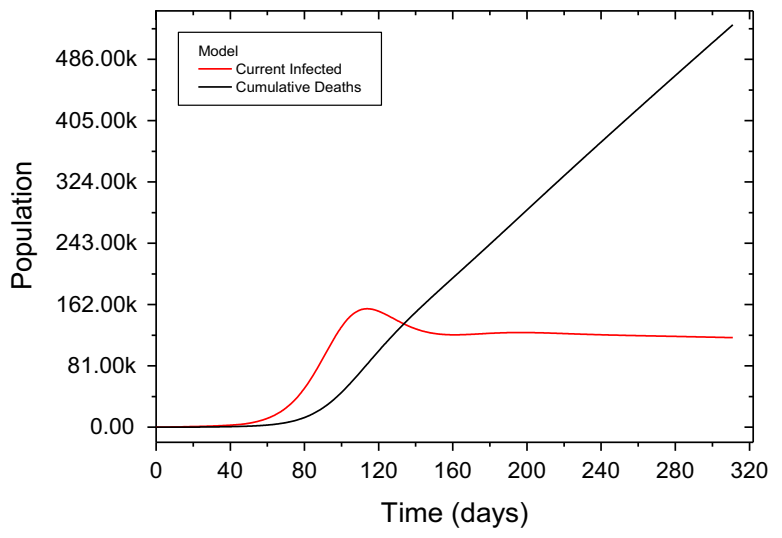

(b)

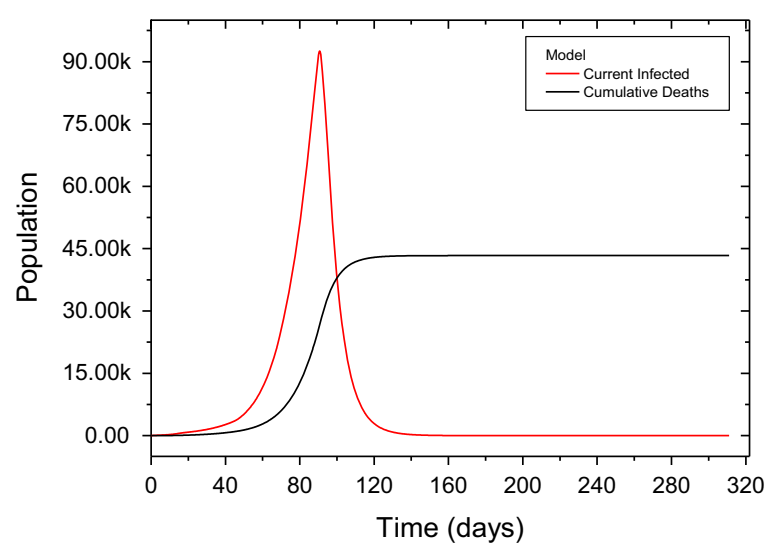

(d)

Fig. 6 Population evolutions considering different social isolation induced by governmental actions: $\mathbf{a} \alpha=0.00$, b $\alpha=0.30$, c $\alpha=0.60, \mathbf{d} \alpha=0.90$

Table 4 Infected and cumulative deaths predicted considering different governmental actions. Hospital infrastructure without any restriction

\begin{tabular}{llll}
\hline $\begin{array}{l}\text { Governmental action } \\
\text { after } 90 \text { days }(\alpha)\end{array}$ & $\begin{array}{l}\text { Peak } \\
(\text { days })\end{array}$ & $\begin{array}{l}\text { Current infected } \\
\text { max. value (pop.) }\end{array}$ & $\begin{array}{l}\text { Cumulative deaths on } \\
\text { December 31, 2020 (pop.) }\end{array}$ \\
\hline 0.00 & 112 & 313,367 & 846,833 \\
0.30 & 114 & 156,440 & 531,503 \\
0.40 & 107 & 114,576 & 399,440 \\
0.50 & 94 & 97,358 & 250,498 \\
0.60 & 92 & 94,473 & 110,509 \\
0.70 & 91 & 93,435 & 61,918 \\
0.80 & 91 & 93,896 & 49,260 \\
0.90 & 91 & 92,561 & 43,331 \\
\hline
\end{tabular}


populations: $\gamma_{\mathrm{C}}$ is associated with the recovered population; $\gamma_{D}$ is related to the death population; and $\gamma_{\mathrm{D}}^{\mathrm{H}}$ is related to the death population inside the group that needs hospital assistance but, due to system collapse, does not receive this assistance. The definition of the fatality rate is based on the relation between cumulative total deaths, $C_{\mathrm{D}}$, and the total cases, $C_{I}$. Therefore, it is possible to use the following expression, considering a similar ratio between the associated rates for the removed population:

$\frac{C_{\mathrm{D}}}{C_{I}}=\frac{\gamma_{\mathrm{D}}}{\gamma_{\mathrm{D}}+\gamma_{\mathrm{C}}} \rightarrow \gamma_{\mathrm{D}}=\left(\frac{C_{\mathrm{D}} / C_{I}}{1-C_{\mathrm{D}} / C_{I}}\right) \gamma_{\mathrm{C}}$.

The recovered parameter, $\gamma_{C}$, is defined based on the period necessary for the immune system response. The death parameter, $\gamma_{\mathrm{D}}$, is defined from the expression presented in Eq. (14). The hospital parameter, $\gamma_{\mathrm{D}}^{\mathrm{H}}$, is defined from the relation of the part of the infected that needs hospital assistance.

In general, the parameter definitions depend on several issues, being a difficult task. The use of step functions confers flexibility to represent different scenarios, meaning that parameters are time dependent. These characteristics are related to the transmission rate and hospital infrastructure, for example.

It is also important to observe that some researches concluded that undocumented novel coronavirus infections are critical for understanding the overall prevalence and pandemic potential of this disease. $\mathrm{Li}$ et al. [13] evaluated Wuhan situation and estimated that $86 \%$ of all infections were undocumented and that

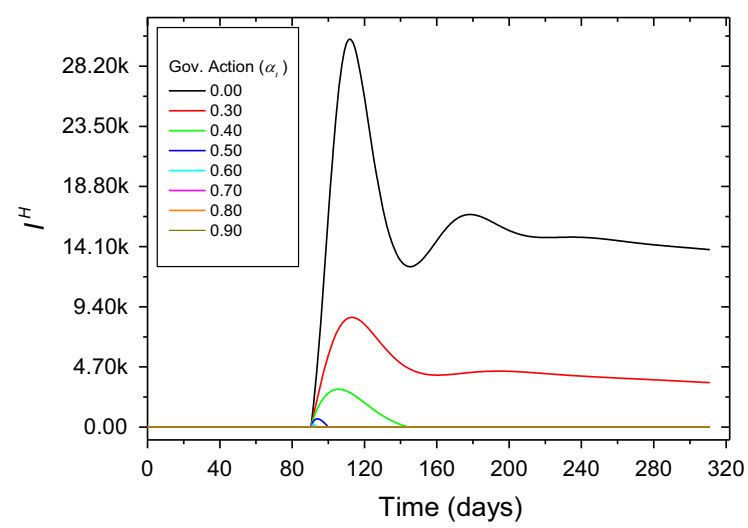

Fig. 7 Population dynamics considering different social isolation induced by governmental actions. Infectious that needs hospital assistance but does not have access due to the lack of infrastructure the transmission rate per person of undocumented infections was $55 \%$ of documented infections. This aspect makes the description even more complex. Table 1 presents the parameters employed for all simulations. Other parameters are adjusted depending on the case.

Numerical simulations are performed considering the fourth-order Runge-Kutta method. A convergence analysis is developed for the presented cases. The next sections treat the COVID-19 dynamics considering two different objectives. Initially, the next section performed a model verification using information from the process experienced by China. Afterward, the subsequent section establishes a model verification of the Brazilian case and investigates different possible scenarios. Simulations are performed in order to clarify the model ability to capture the main characteristics of the COVID-19 dynamics.

\section{Model verification}

A model verification is carried out using information available on Worldometer [23]. Once again, it should be pointed out that the analysis is based on average populations, assumed to have spatial homogeneous distribution. In principle, it would be more realistic to represent small areas where it is expected homogeneous response. Nevertheless, the consideration of the whole country is useful for a general qualitative behavior of the outbreak. China data are considered as the benchmark case due to the fact that it is the first case in the world and can be used to gather important information to support the predictions for other countries. This analysis is employed to establish a comparison with real data. Table 2 presents additional parameters employed for the simulations. They are based on the information of the Lin et al. [15] that, in turn, is based on other references as He et al. [11] and Breto et al. [3]. Furthermore, an average value of the fatality rate $\frac{C_{\mathrm{D}}}{C_{I}}=0.02$ is adopted based on China actual data. It is important to highlight that this value is calculated with the available data and the existence of unreported cases can substantially change this number. For more details, see other citations referenced therein.

Parameters presented in Tables 1 and 2 are employed for simulations with a population of 


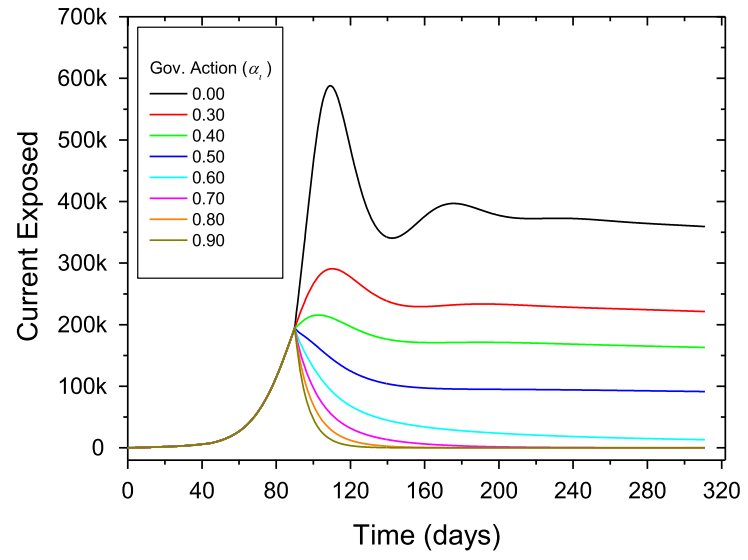

(a)

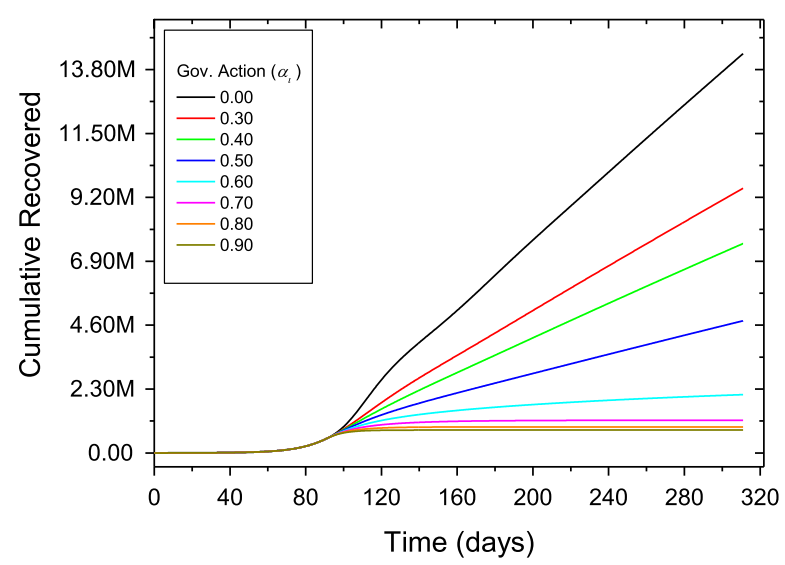

(c)

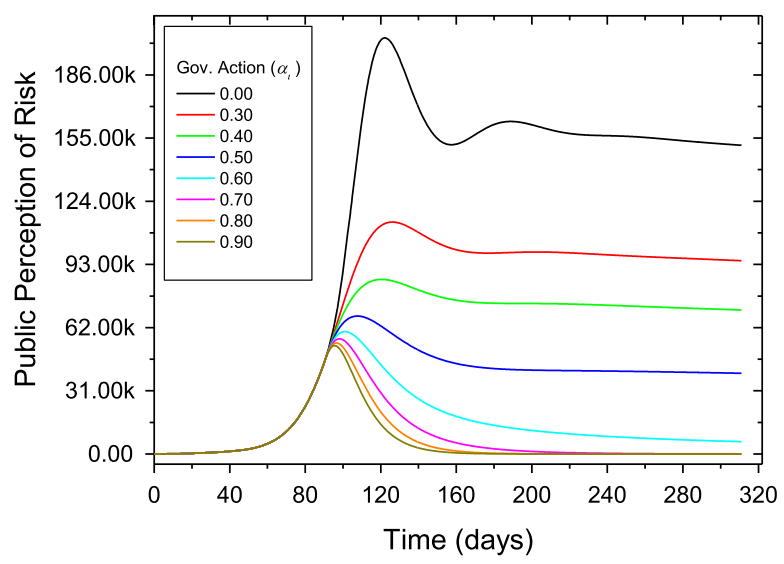

(e)

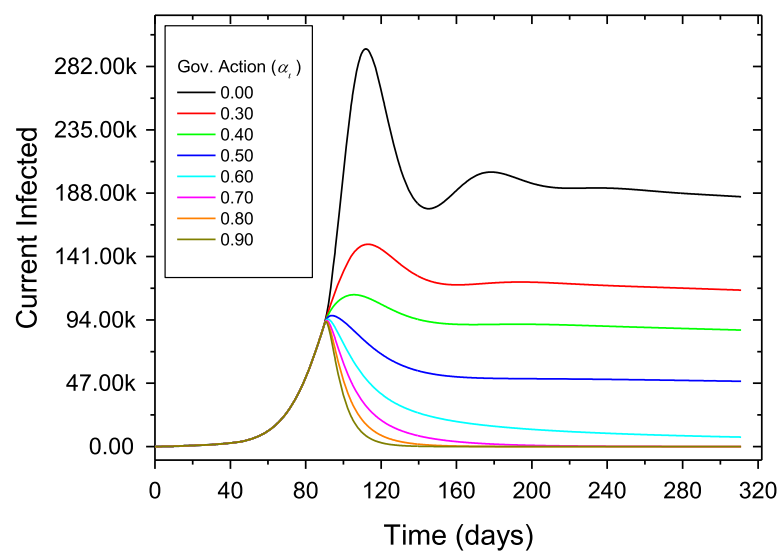

(b)

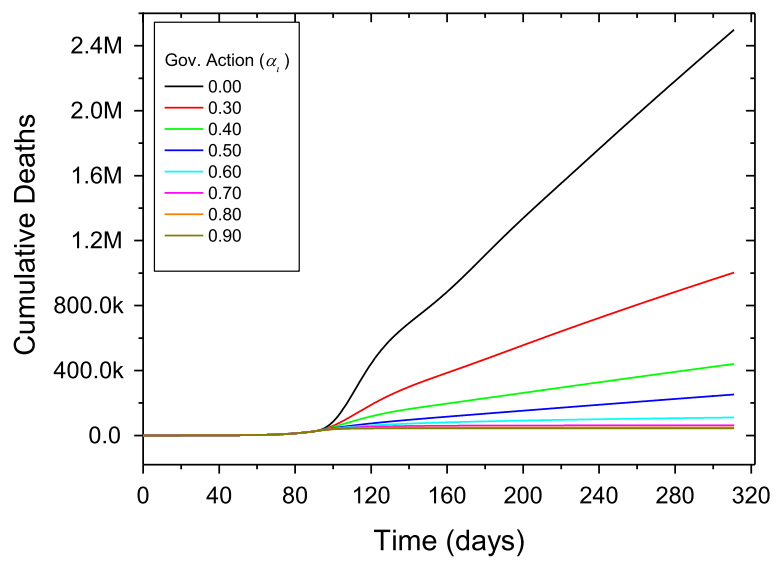

(d)

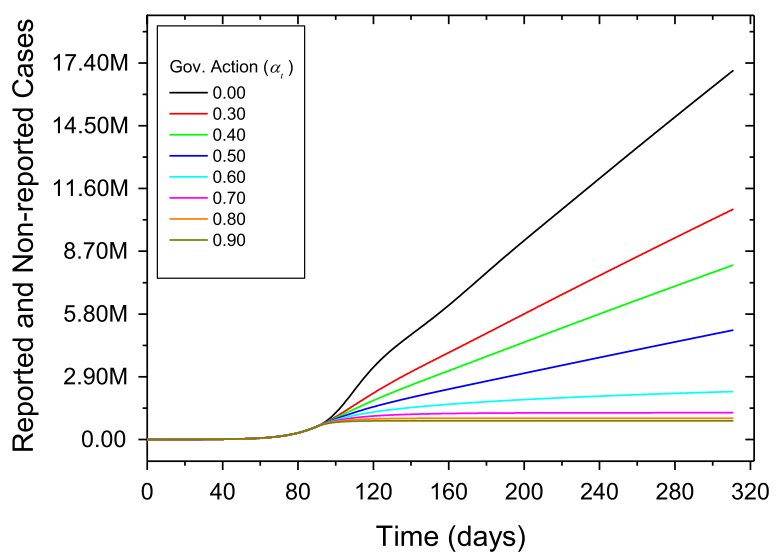

(f)

Fig. 8 Population dynamics considering different social isolation induced by governmental actions: a current exposed, b current infected, $\mathbf{c}$ cumulative recovered, $\mathbf{d}$ cumulative deaths, $\mathbf{e}$ public perception risk and $\mathbf{f}$ reported and non-reported cases 

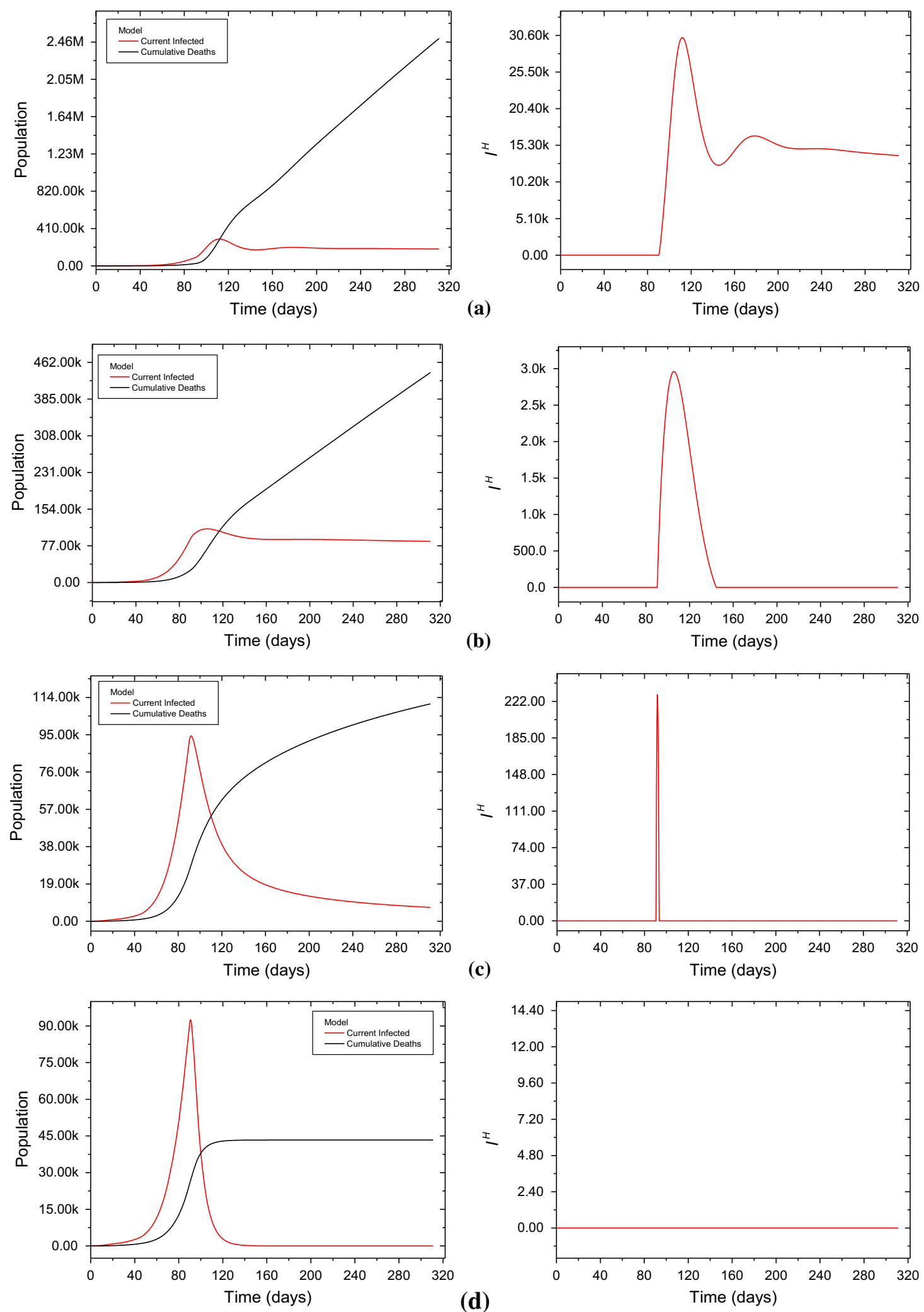
४Fig. 9 Population evolutions considering different transmission rates, altered by governmental actions: a $\alpha=0.00$, $\mathbf{b} \alpha=0.40, \mathbf{c} \alpha=0.60, \mathbf{d} \alpha=0.90$

$N=1.43$ billion and an initial state with 554 infected persons $\left(I_{0}=554\right)$, relative to January 22 , 2020. In addition, susceptible population initial condition is assumed to be $S_{0}=0.9 \mathrm{~N}$. Another information needed for the model is the number of exposed persons for each infected person. It is assumed that each infected person has the potential to expose 20 persons, $E_{0}=20 I_{0}$.

Hospital infrastructure is considered to be without any restriction, which means that all the population that needs assistance is assisted, and therefore, population $I^{\mathrm{H}}=0$ for all time during the whole simulation. Figure $3 \mathrm{a}$ presents the infected and cumulative death populations showing a good agreement between simulation and real data obtained from Worldometer [23]. Figure $3 b$ presents the evolution of other variables of the model. Note that, due to chronological issues, the whole cycle is observed on Chinese data, defining a peak-vanish pattern associated with an increase in the infectious followed by a decrease to low levels. Based on that, it is possible to say that the model is capable to describe the whole cycle of COVID-19 dynamics.

\section{Brazilian case}

This section has the objective to investigate COVID19 dynamics in Brazil. Initially, a model calibration is performed, establishing a model verification for Brazil. In the sequence, numerical simulations are carried out investigating different scenarios. All simulations consider a population of $N=209.3$ million and an initial state with 1 infected $\left(I_{0}=1\right)$ and 250 exposed persons $\left(E_{0}=250\right)$, relative to February 25, 2020. Parameters listed in Tables 1 and 3 are employed in all the simulations [9, 17]. An average value of fatality rate $\frac{C_{\mathrm{D}}}{C_{I}}=0.05$ is adopted based on Brazil actual data. Values adopted for $\alpha_{i}$ consider three moments associated with governmental action $(0,15$ and 45 days), representing that the effect of two events that occurred from the moment of the first infected person was identified and the present moment when the paper is being written. In the first event, which occurred after 15 days, some regions of the country implemented actions of social isolation, such as closing the schools/universities and adopting remote work. One month after, there was a relaxation of the social isolation, which has been maintained until May 2020.

Figure $4 \mathrm{a}$ presents the infected population and cumulative deaths evolution obtained from numerical simulations and real data obtained from Worldometer [23], showing that the same trend of the other cases is followed, being enough to have a general scenario. Figure $4 \mathrm{~b}$ presents the evolution of other variables of the model.

\subsection{Different scenarios}

Different scenarios are now investigated considering a period of time until the end of 2020. Brazilian

Table 5 Infected and cumulative deaths predicted considering different governmental actions

\begin{tabular}{lllrr}
\hline $\begin{array}{l}\text { Governmental intervention } \\
\text { after } 90 \text { days }(\alpha)\end{array}$ & $\begin{array}{l}\text { Current infected- } \\
\text { peak (days) }\end{array}$ & $\begin{array}{l}\text { Current infected- } \\
\text { max. value (pop.) }\end{array}$ & $\begin{array}{l}\text { Cumulative deaths on } \\
\text { December 31, 2020 (pop.) }\end{array}$ & $\begin{array}{l}I^{\mathrm{H}} \text {-lack of hospital } \\
\text { assistance (pop.) }\end{array}$ \\
\hline 0.00 & 112 & 294,996 & $2,498,629$ & 30,310 \\
0.30 & 113 & 150,090 & $1,003,322$ & 8575 \\
0.40 & 106 & 112,670 & 440,749 & 2962 \\
0.50 & 94 & 97,210 & 252,369 & 643 \\
0.60 & 92 & 94,452 & 110,715 & 229 \\
0.70 & 91 & 93,432 & 61,948 & 76 \\
0.80 & 91 & 92,896 & 49,260 & 0 \\
0.90 & 91 & 92,561 & 43,331 & 0 \\
\hline
\end{tabular}




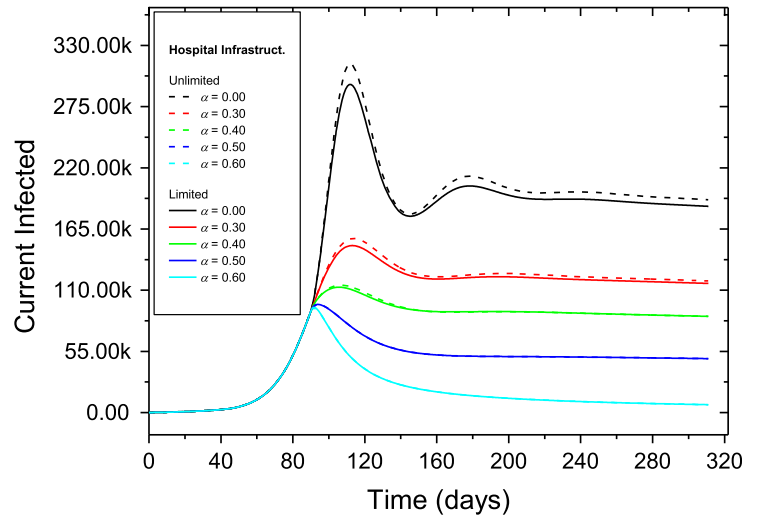

(a)

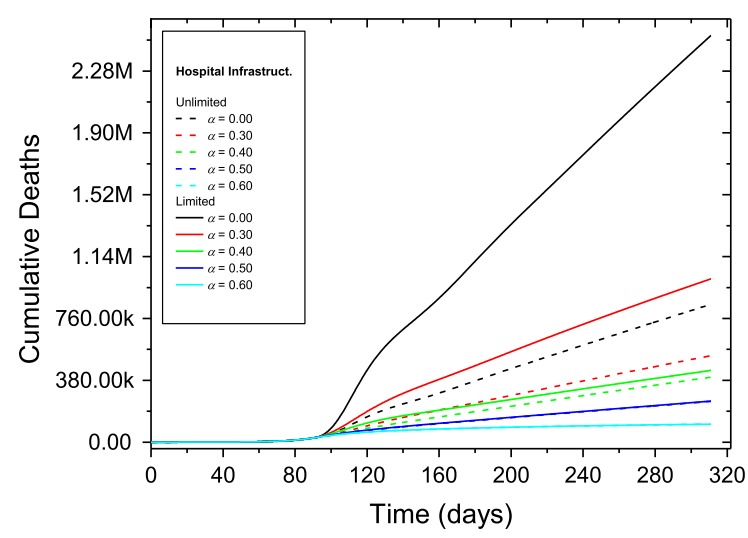

(b)

Fig. 10 Influence of the hospital infrastructure limitations on the population dynamics considering different social isolation induced by governmental actions: a current infected, b cumulative deaths

governmental action has the characteristic to be without a central coordination that makes the social isolation a polemic point, different from the great majority of the world. Based on that characteristic, it is important to present simulations showing distinct scenarios related to social isolation. Figure 5 presents different scenarios defined by the transmission rate induced by governmental actions represented by different values of the parameter $\alpha$, showing the evolution of all populations involved on COVID-19 dynamics. It is assumed an unlimited hospital infrastructure, which means that all the population that needs assistance is assisted. Calibrated values are maintained for the period of the first 90 days, defined from the available data for the present moment when the paper is being written. From this point forward, the future is predicted considering different values of $\alpha$,

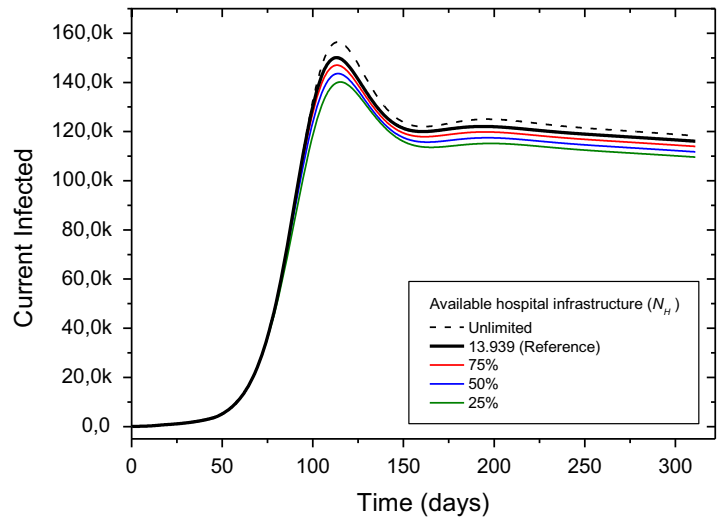

(a)

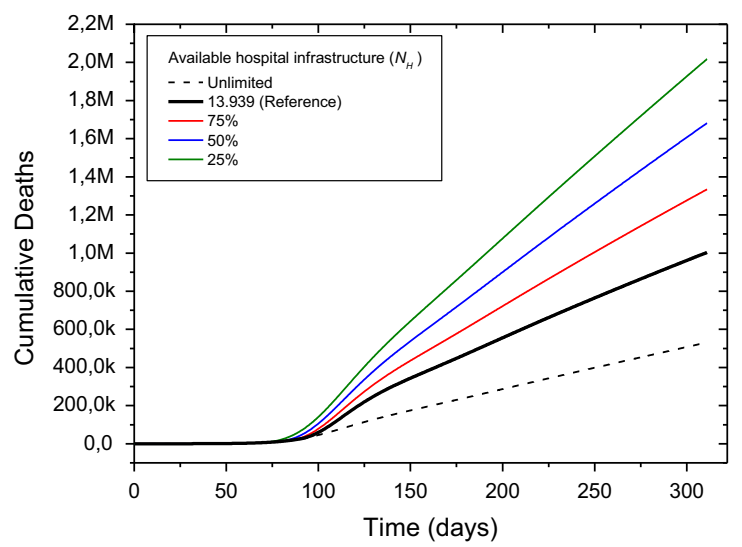

(b)

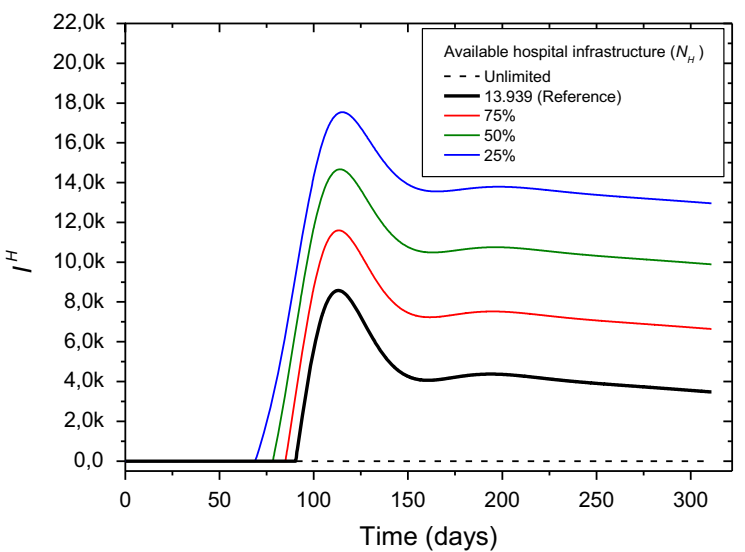

(c)

Fig. 11 Population dynamics considering different hospital infrastructure: a current infected, b cumulative deaths and c infectious that needs hospital assistance but does not have access due to the lack of infrastructure 


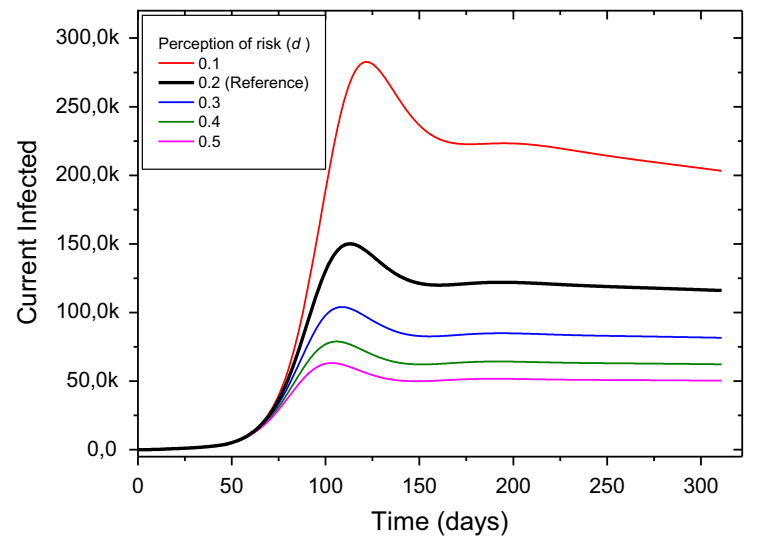

(a)

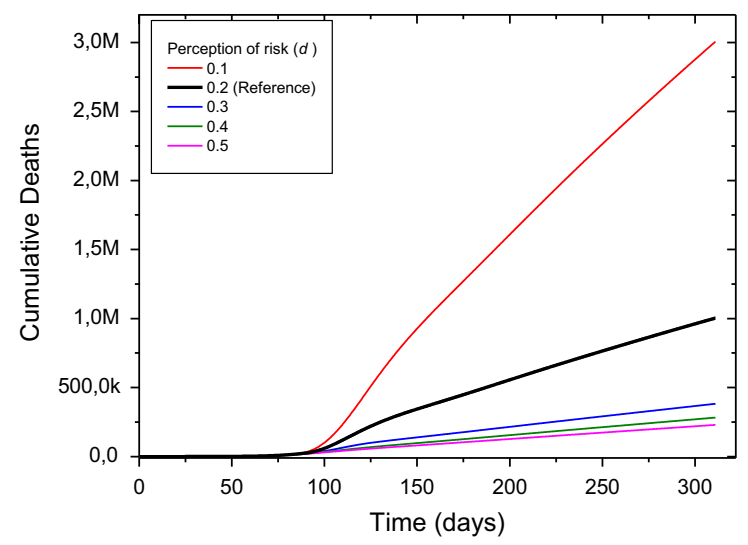

(b)

Fig. 12 Influence of the perception of risk represented by parameter $d$ on the population dynamics: a current infected, b cumulative deaths

which characterizes distinct scenarios. The following parameter values are adopted: 0.00, 0.30, 0.40, 0.50, $0.60,0.70,0.80$ and 0.90 . These scenarios represent different conditions associated with fixed governmental actions adopted at the end of the first 90 days that are maintained until the end of the year. It should be noticed that the curves have dramatic different values, and therefore, the number of infected and deaths presents huge differences. There is a huge reduction in both numbers with the increase in social isolation. In addition, there is an important qualitative change related to the infectious population. The social isolation produces an infectious dynamics with a peak followed by a decrease to small numbers, called peakvanish case, as shown in Fig. $5 \mathrm{~b}$ for $\alpha$ between 0.60 and 0.90 . On the other hand, the lack of social isolation produces a curve with a plateau characteristic

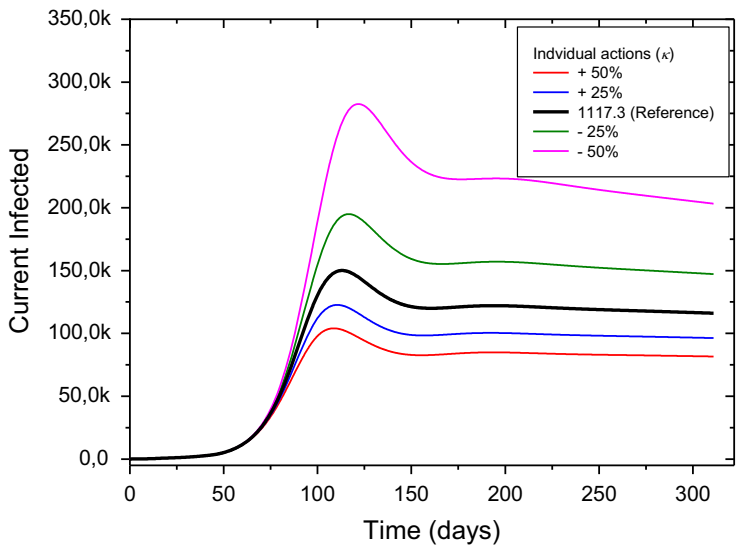

(a)

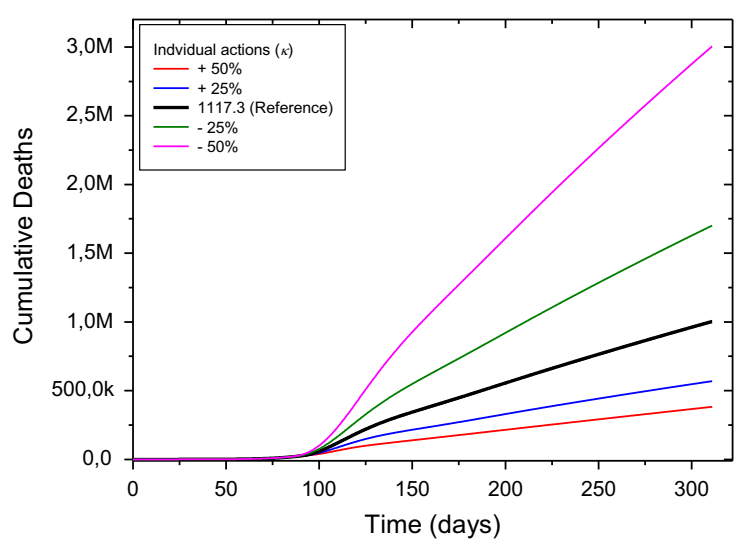

(b)

Fig. 13 Influence of the individual actions represented by parameter $\boldsymbol{\kappa}$ on the population dynamics: a current infected, b cumulative deaths

observed in Fig. 5b for $\alpha$ between 0.00 and 0.50 , which means that the critical period is spread over the time.

Figure 6 presents the detailed views of the dynamics of populations of current infected and cumulative deaths highlighting some characteristic behaviors. Once again, it is evident that the number of involved populations is dramatically different for each kind of governmental action. The difference of the two possible behaviors, characterized by the peak-vanish and plateau behaviors for the current infected populations, is also observed.

Based on these scenarios, it should be pointed out that different governmental actions related to social isolation effect result in dramatically different numbers of infected and cumulative deaths. Table 4 
Table 6 Different scenarios considering several approaches of the governmental actions implementation

\begin{tabular}{clllllllllll}
\hline Case & \multicolumn{1}{l}{$T_{\text {Gov }}^{(i)}$} & \multicolumn{1}{c}{10} & & & & & & & \\
\cline { 2 - 10 } & 0 & 15 & 45 & 90 & 120 & 150 & 180 & 210 & 240 & 270 & 300 \\
\hline$\alpha_{i}$ & & & & & & & & & & & \\
1 & 0.00 & 0.40 & 0.30 & 0.70 & 0.50 & 0.70 & 0.50 & 0.70 & 0.50 & 0.70 & 0.50 \\
2 & 0.00 & 0.40 & 0.30 & 0.70 & 0.70 & 0.50 & 0.50 & 0.70 & 0.70 & 0.50 & 0.50 \\
3 & 0.00 & 0.40 & 0.30 & 0.80 & 0.30 & 0.80 & 0.30 & 0.80 & 0.30 & 0.80 & 0.30 \\
4 & 0.00 & 0.40 & 0.30 & 0.80 & 0.40 & 0.80 & 0.40 & 0.80 & 0.40 & 0.80 & 0.40 \\
5 & 0.00 & 0.40 & 0.30 & 0.80 & 0.50 & 0.80 & 0.50 & 0.80 & 0.50 & 0.80 & 0.50 \\
6 & 0.00 & 0.40 & 0.30 & 0.80 & 0.70 & 0.60 & 0.50 & 0.40 & 0.30 & 0.30 & 0.30 \\
\hline
\end{tabular}

Interventions prior to 90 days preserved

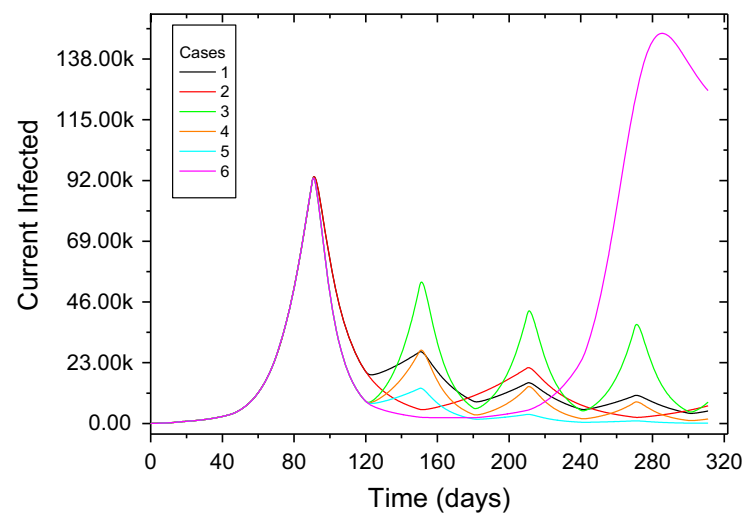

(a)

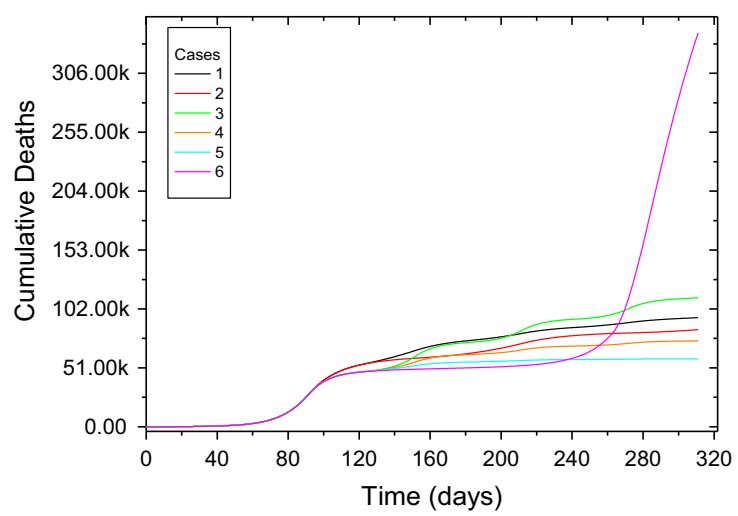

(b)

Fig. 14 Population dynamics considering different social isolation induced by governmental actions: a current infected, b cumulative deaths

summarizes the results showing that a worst scenario of 846,833 deaths is in huge contrast with the best scenario of 43,331 deaths. This comparison clearly indicates that a more appropriate approach can result in a huge number of preserved lives.

\subsection{Influence of hospital infrastructure}

One of the most relevant points related to COVID-19 evolution is the hospital infrastructure. Based on that, different scenarios are now of concern estimating distinct hospital infrastructure levels. The constraints are difficult to be quantified since it is not only the number of hospital beds available, but medical staff, drug availability and medical equipment are also necessary to define the hospital infrastructure. The absence of this infrastructure increases the number of deaths since the population that needs assistance does not receive it. The specific infrastructure for this population is represented in the model by the total number of available intensive care units (ICUs), designed by $N_{\mathrm{H}}$. Data accessed from the Brazilian Ministry of Health [1,2] show that Brazil has close to 40,000 ICUs. Nevertheless, only 13,939 are eligible for the treatment of patients with COVID-19. Numerical simulations are carried out considering the value of $N_{\mathrm{H}}$. It is important to highlight that there is a nonhomogeneous geographic distribution in Brazil, with the ratio of 9 and 21 ICU beds per 100,000 inhabitants in the north and southeast regions of the country, respectively $[6,7]$. In addition, some hospitals suffer with a lack of health professionals to assist patients with COVID-19. This condition can make the lack of ICUs even more critical.

Figure 7 shows the evolution of the population that needs hospital assistance and does not receive it considering different scenarios defined for the distinct governmental actions treated on the previous simulations. Figure 8 presents the evolution of all populations involved on COVID-19 dynamics. In general, it is noticeable the same qualitative behavior observed for the populations in the previous simulations for all 
Table 7 Infected and cumulative deaths predicted considering different governmental actions associated with cyclic actions and hardening action followed by a progressive softening action

\begin{tabular}{lllll}
\hline $\begin{array}{l}\text { Governmental intervention } \\
\text { after } 90 \text { days }(\alpha)\end{array}$ & $\begin{array}{l}\text { Current infected- } \\
\text { peak (days) }\end{array}$ & $\begin{array}{l}\text { Current infected- } \\
\text { max. value (pop.) }\end{array}$ & $\begin{array}{l}\text { Cumulative deaths on } \\
\text { December 31, 2020 (pop.) }\end{array}$ & $\begin{array}{l}I^{\mathrm{H}}-\text { lack of hospital } \\
\text { assistance (pop.) }\end{array}$ \\
\hline Case 1 & 91 & 93,432 & 94,520 & 76 \\
Case 2 & 91 & 93,432 & 84,111 & 76 \\
Case 3 & 91 & 92,896 & 111,845 & 0 \\
Case 4 & 91 & 92,896 & 74,448 & 0 \\
Case 5 & 91 & 92,896 & 58,831 & 0 \\
Case 6 & $91 / 286^{\mathrm{a}}$ & $92,896 / 147,700^{\text {a }}$ & 340,934 & 8216 \\
\hline
\end{tabular}

${ }^{\mathrm{a}}$ Second wave peak

cases, but two important points should be observed: The size of the populations is totally different, which means that the infected populations and deaths are completely different; the other important point to be observed is related to the hospital infrastructure. Note that there is a dramatic difference in terms of necessary hospital infrastructure, either for the number of hospital space or the spread over the time. The decrease in the social isolation is associated with the increase in the infected and death populations. In addition, infectious population presents an important plateau behavior that is related to an increase in deaths. Once again, it can be observed that different governmental actions result in dramatically different numbers of infected and cumulative deaths.

Figure 9 highlights some characteristic behaviors of the dynamics. The left panel shows the populations of current infected and cumulative deaths, whereas the right panel shows the population that needs hospital assistance and does not receive it. As for the previous cases, the number of involved populations is dramatically different for each kind of governmental action, and two possible behaviors, characterized by the peak-vanish and plateau behaviors for the current infected populations, are also observed.

Table 5 summarizes the results showing a worst scenario of 2.5 million deaths (close to $1.2 \%$ of the Brazilian population) and a best scenario of 43,331 deaths, an even more dramatic difference when the hospital infrastructure is incorporated into the analysis. Results show that the limitations of the hospital infrastructure cause more than 30,000 infected individuals inside the group that needs hospital assistance maybe left without access, a condition associated with a high fatality rate.

Figure 10 shows a comparison between numerical results considering two situations relative to the specific hospital infrastructure required to deal with the COVID-19. The first set of results (Unlimited Hosp. Infrastructure) considers an ideal condition where there is no restriction of the hospital infrastructure to assist part of the infectious that needs hospital assistance, whereas the second one (Limited Hosp. Infrastructure) considers situations where restrictions are defined by the total number of available intensive care units (ICUs). Numerical results confirm that the absence of this infrastructure largely increases the number of deaths since the population that needs assistance does not receive it. This increase in the deaths from 846,833 to $2,498,629$ (for $\alpha=0.00$ after 90 days) is an emblematic situation associated with an increase of more than $200 \%$.

Hospital infrastructure can be altered by different ways. It is possible to increase this creating field hospitals, but it is also possible to decrease this number due to loss of medical staff or equipment. Mathematically speaking, this effect can be represented in the model parameter $N_{\mathrm{H}}$. In this regard, a simulation is carried out considering different values of this parameter, established from percentages of the original hospital infrastructure. Figure 11 presents results considering unlimited hospital infrastructure and some different levels of coverage for patients with COVID-19 that need ICU treatment: 100\%, 75\%, 50\% and $25 \%$. The scenario of $\alpha=0.30$ after the first 90 days is considered, and the reference number of 

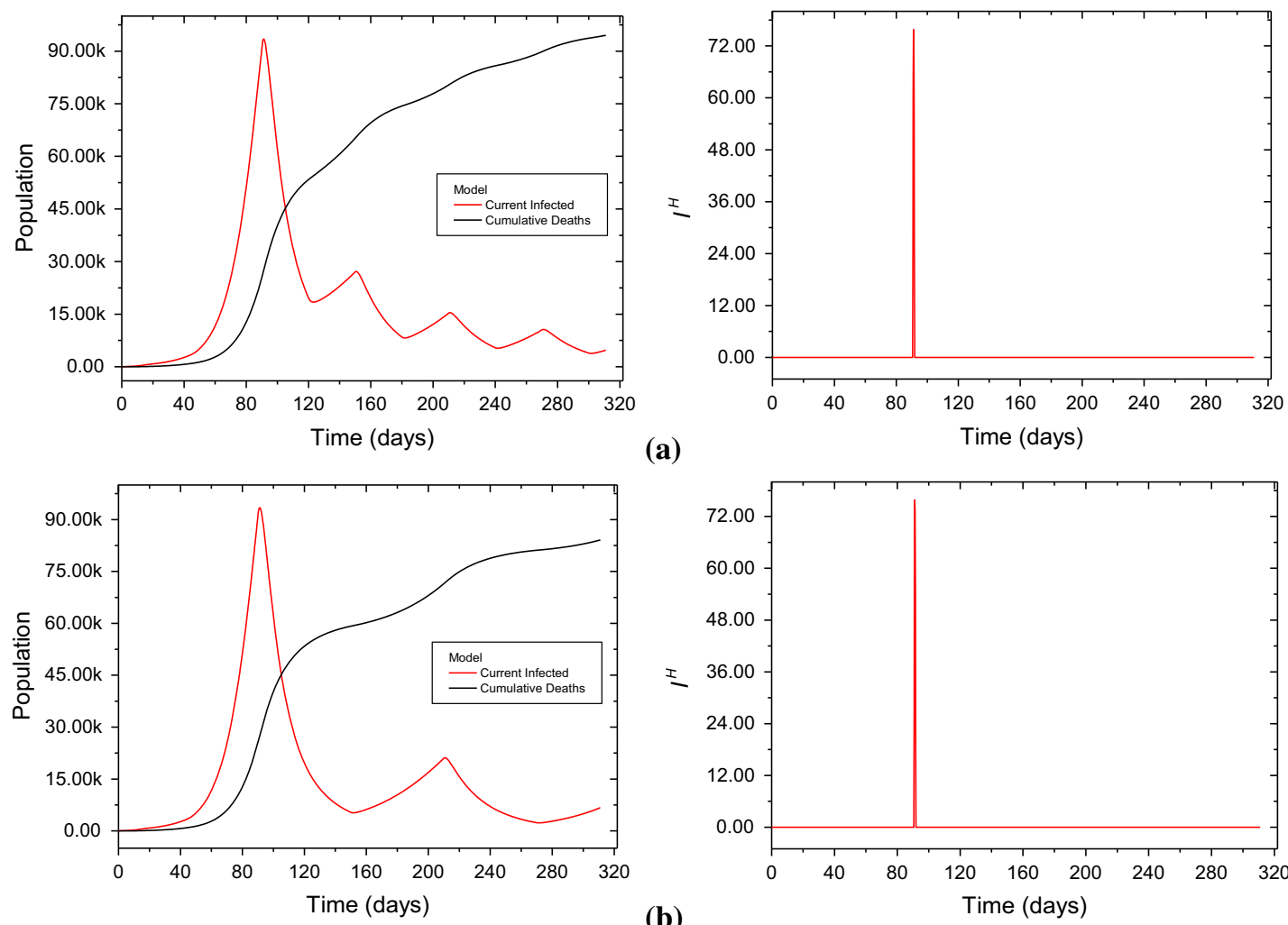

(a)
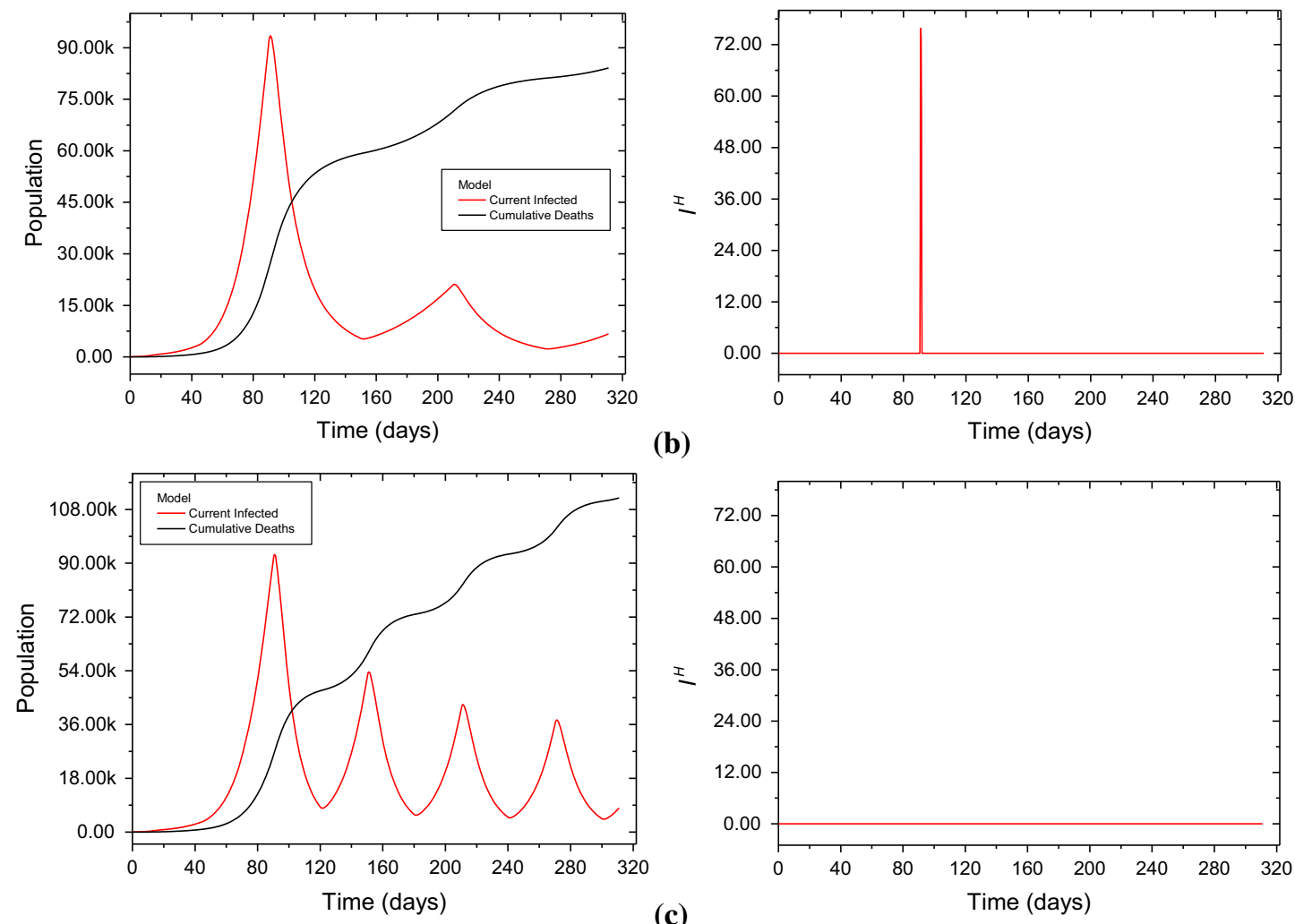

(b)
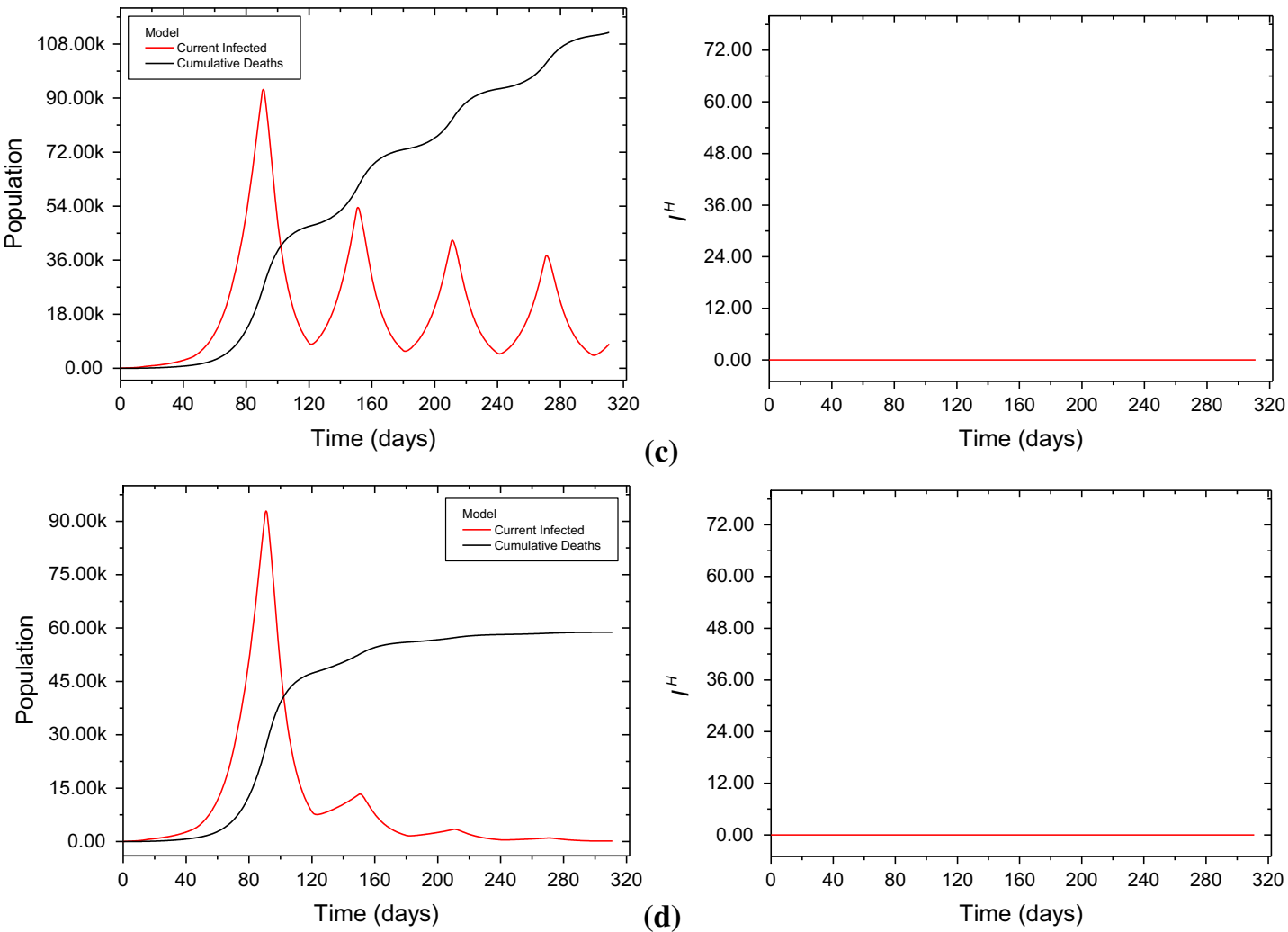

(c)

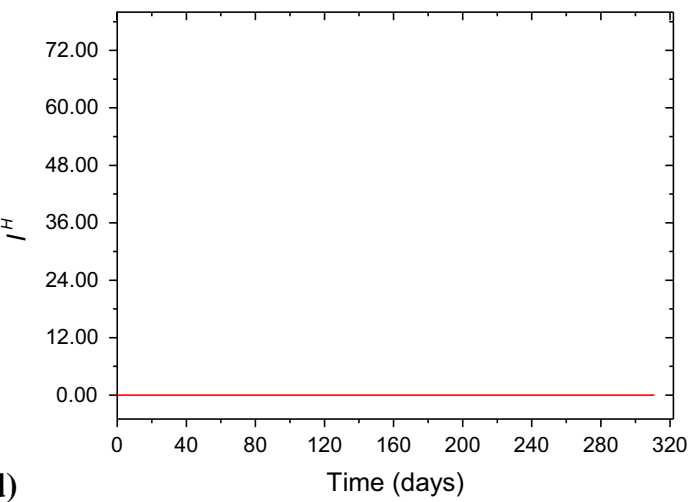


4Fig. 15 Population evolutions considering different transmission rates, altered by cyclic governmental actions: a Case 1, b Case 2, c Case 3, and e Case 5

13,939 ICUs eligible for treatment is adopted. Current infected, cumulative deaths and the part of the infectious that needs hospital assistance but does not have access due to the lack of infrastructure, $I^{\mathrm{H}}$, are shown illustrating how the reduction in the hospital infrastructure impacts the disease dynamics. The presented scenarios show a persistent crisis on the hospital infrastructure characterized by the plateaus pattern.

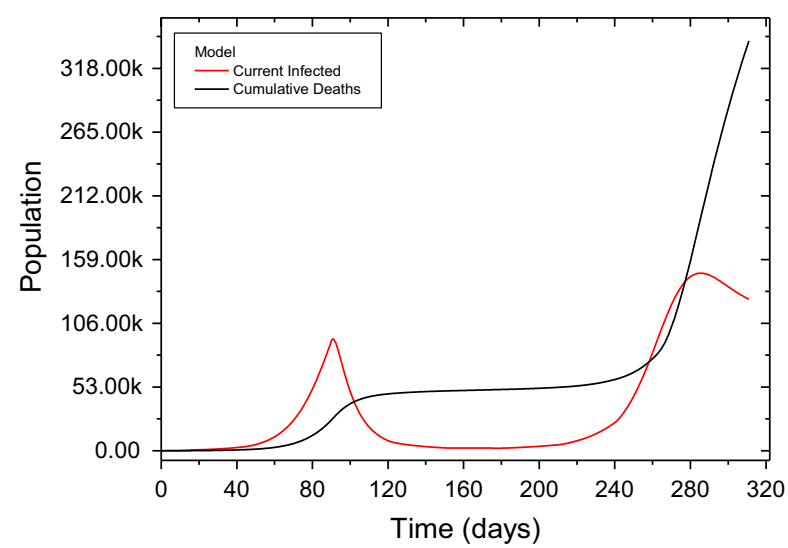

4.3 Influence of the perception risk and individual actions

Since social isolation is the essential point that defines the COVID-19 dynamics, two key points need to be considered: perception of risk, associated with individual actions; and governmental actions, which force social isolation. The analysis of the perception of risk, represented by parameter $d$, is now in focus. Different scenarios are evaluated considering several possible values of this parameter. Figure 12 presents results related to simulations of distinct values of the perception risk parameter, showing current infected population and cumulative deaths. The scenario for $\alpha=0.30$ used in the previous section is considered. It is observed that the increase in this parameter reduces

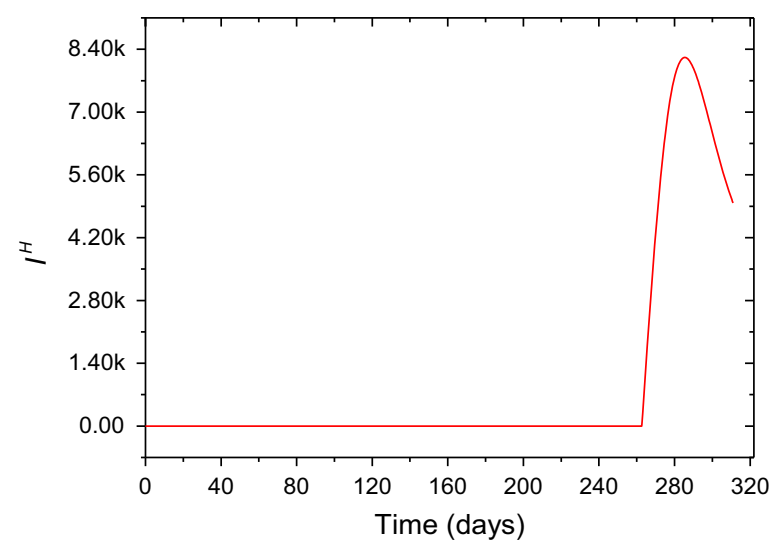

Fig. 16 Population evolutions considering different transmission rates, altered by a governmental hardening action followed by a progressive softening action: Case 6. Interventions prior to 90 days preserved

Table 8 Different scenarios considering different early governmental actions approaches: Constant, Progressive and Cyclic

\begin{tabular}{|c|c|c|c|c|c|c|c|c|c|c|c|c|}
\hline \multirow[t]{2}{*}{ Approach } & \multirow[t]{2}{*}{$\alpha_{2}$} & \multicolumn{11}{|l|}{$T_{\text {Gov }}^{(i)}$} \\
\hline & & 0 & 15 & 45 & 90 & 120 & 150 & 180 & 210 & 240 & 270 & 300 \\
\hline \multicolumn{13}{|l|}{$\alpha_{i}$} \\
\hline \multirow[t]{3}{*}{ Constant } & 0.70 & 0.00 & 0.70 & 0.70 & 0.70 & 0.70 & 0.70 & 0.70 & 0.70 & 0.70 & 0.70 & 0.70 \\
\hline & 0.80 & 0.00 & 0.80 & 0.80 & 0.80 & 0.80 & 0.80 & 0.80 & 0.80 & 0.80 & 0.80 & 0.80 \\
\hline & 0.90 & 0.00 & 0.90 & 0.90 & 0.90 & 0.90 & 0.90 & 0.90 & 0.90 & 0.90 & 0.90 & 0.90 \\
\hline \multirow[t]{3}{*}{ Progressive } & 0.70 & 0.00 & 0.70 & 0.60 & 0.50 & 0.50 & 0.50 & 0.50 & 0.50 & 0.50 & 0.50 & 0.50 \\
\hline & 0.80 & 0.00 & 0.80 & 0.70 & 0.60 & 0.50 & 0.50 & 0.50 & 0.50 & 0.50 & 0.50 & 0.50 \\
\hline & 0.90 & 0.00 & 0.90 & 0.80 & 0.70 & 0.60 & 0.50 & 0.50 & 0.50 & 0.50 & 0.50 & 0.50 \\
\hline \multirow[t]{3}{*}{ Cyclic } & 0.70 & 0.00 & 0.70 & 0.70 & 0.50 & 0.50 & 0.70 & 0.70 & 0.50 & 0.50 & 0.70 & 0.70 \\
\hline & 0.80 & 0.00 & 0.80 & 0.80 & 0.50 & 0.50 & 0.80 & 0.80 & 0.50 & 0.50 & 0.80 & 0.80 \\
\hline & 0.90 & 0.00 & 0.90 & 0.90 & 0.50 & 0.50 & 0.90 & 0.90 & 0.50 & 0.50 & 0.90 & 0.90 \\
\hline
\end{tabular}


Table 9 Infected and cumulative deaths predicted considering different early governmental actions approaches: Constant, Progressive and Cyclic

\begin{tabular}{lllll}
\hline Approach & $\begin{array}{l}\text { Governmental } \\
\text { actions }(\alpha)\end{array}$ & $\begin{array}{l}\text { Current infected- } \\
\text { peak (days) }\end{array}$ & $\begin{array}{l}\text { Current infected- } \\
\text { max. value (pop.) }\end{array}$ & $\begin{array}{l}\text { Cumulative deaths } \\
\text { on December 31, 2020 (pop.) }\end{array}$ \\
\hline Constant & 0.70 & 18 & 636 & 469 \\
& 0.80 & 17 & 614 & 259 \\
Progressive & 0.90 & 17 & 602 & 191 \\
& 0.70 & $18 / 311^{\mathrm{a}}$ & $635 / 51,386^{\mathrm{a}}$ & 11,969 \\
& 0.80 & $17 / 311^{\mathrm{a}}$ & $614 / 11,969^{\mathrm{a}}$ & 196 \\
Cyclic & 0.90 & 17 & 602 & 636 \\
& 0.70 & 18 & 614 & 602 \\
\end{tabular}

${ }^{\mathrm{a}}$ No peak-value in the 311 day

the infectious populations and, consequently, the number of deaths.

Figure 13 presents results related to the effect of individual actions, represented by parameter $\kappa$. Numerical simulations are developed considering the variation of the parameter value from -50 to $+50 \%$ with respect to the reference value of $\kappa=1117.3$ listed in Table 1 and adopted for all the other simulations. Results show that higher values reduce the current infected and cumulative deaths.

\subsection{Influence of the governmental action}

Different scenarios are now evaluated considering several possible implementations of governmental actions. Table 6 presents cases where the previous adopted values of $\alpha$ for the interventions prior to 90 days are maintained and future actions are implemented considering a combination of hardening/softening governmental actions. Results from the previous analysis show that a minimum value of $\alpha=0.70$ must be adopted at $T_{\text {Gov }}^{(3)}=90$ days to maintain the number of deaths below 100,000. Therefore, this minimum value is adopted for the analysis.

Several possibilities for the social isolation have been discussed worldwide. One promising scenario involves the combination of triggering hardening/softening actions [9].

Figure 14 shows the population evolutions considering Cases 1-6. Cases 1-5 are associated with cyclic governmental actions. These actions result in multiple subsequent infection waves. Case 6 represents a condition where a governmental hardening action is implemented after 90 days, followed by a progressive softening action. For this situation, the increase in the infectious population during the second wave, together with the limitations of the available hospital infrastructure, results in more than 8000 infected individuals within the group who needs hospital assistance. This contributes to a larger number of deaths that could be avoided. These scenarios show that social isolation combined with proper hospital infrastructure can drastically reduce the number of deaths.

Table 7 summarizes these results, showing a worst scenario of 340,934 deaths and a best scenario of 58,831 deaths, indicating that a reduction in the number of deaths is possible with a proper isolation strategy controlled by governmental action.

Figure 15 highlights some characteristic behaviors of the population dynamics of the current infected and cumulative deaths for some cases associated with cyclic governmental actions, whereas Fig. 16 presents results for a hardening action followed by a progressive softening action. The left panel shows the populations of the current infected and cumulative deaths, whereas the right panel shows the population that needs hospital assistance and does not receive it. Results show multi-peak behavior followed by vanish or plateau behaviors for the current infected populations. 

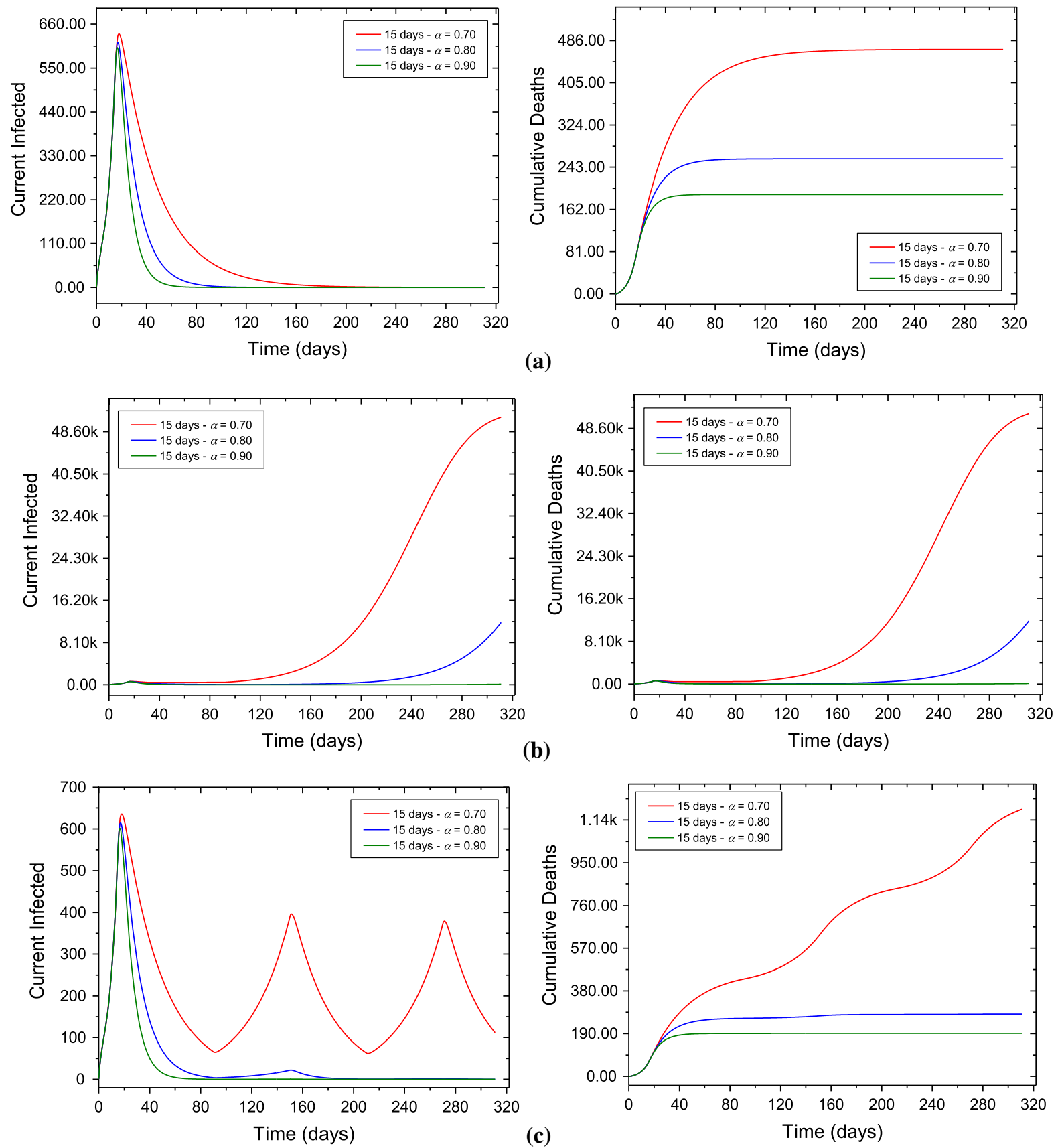

Fig. 17 Evolution of the infected and cumulative deaths populations considering different early governmental actions approaches implemented at the beginning of the pandemic in

Brazil: a Constant, b Progressive and c Cyclic. Early governmental intervention (prior to 90 days)

As a highly contagious disease, COVID-19 requires the implementation of governmental actions in the very beginning. Previous analysis has shown the difficulties to control or reduce the number of infectious and deaths if nothing is done at early stages. With

the objective to analyze the importance of the implementation of a rapid response, three approaches involving early governmental actions are considered: Constant, Progressive and Cyclic. In the first one, a constant action is adopted from the beginning of the 
intervention; the second considers a progressive reduction in the action until a value of 0.50 is reached; and the third one considers a cyclic variation between two levels of action with a period of two months. For all the cases, the governmental action begins in the 15th day. Table 8 presents the cases description, and Table 9 summarizes results for the three approaches. Due to the early actions taken, none of the 9 cases presented a condition of lack of hospital infrastructure for the part of the infectious population requiring specific assistance. Figure 17 presents the evolution of the current infected (left panel) and the cumulative deaths (right panel).

These results show that, in comparison with previous cases, the use of early governmental actions causes a smaller population of infectious, for which the cumulative deaths can be below 200. There is also no shortage of hospital infrastructure for the part of the infectious population requiring assistance. Overall, the Constant approach presents the best results associated with a smaller number of cumulative deaths at the end of the period, but with the cost of maintaining a severe level of social isolation during a long period of time. The use of an initial $\alpha$ value of 0.90 results in a similar behavior for the three approaches, with a number of cumulative deaths lower than 200. For initial values of $\alpha$ of 0.70 and 0.80 , the Progressive approach reveals the effect of a second wave (as in Case 6 of the previous analysis), resulting in a large number of deaths. A similar behavior is observed considering the Cyclic approach for $\alpha=0.70$, where many deaths are observed due to a second and a third infectious waves. However, for $\alpha=0.80$ the Cyclic approach furnishes lower values of cumulative deaths, similar to the constant approach. Therefore, it can be an interesting alternative to replace the constant approach with the advantage of imposing a less severe governmental action and, therefore, a condition of less severe social isolation over the whole period.

It is important to highlight that, due to the strong sensitivity of the system nonlinear dynamics, small changes in conditions or control parameters can greatly affect the evolution of populations. Therefore, success in controlling the pandemic and reducing deaths depends on the adoption of approaches and mechanisms that allow for monitoring the evolution of populations together with the rapid implementation of control procedures in the form of efficient governmental actions. COVID-19 nonlinear dynamics has rich responses, and a proper comprehension of system dynamics is essential for a proper scenario management.

\section{Conclusions}

A mathematical model based on the susceptibleexposed-infectious-recovered framework is employed to describe the COVID-19 evolution. The proposed model considers the following novel aspects: The removed populations are composed of recovered and death populations; population that needs hospital assistance is incorporated allowing the analysis of the lack of hospital infrastructure. A benchmark case is treated considering available data from China. Afterward, Brazilian case is analyzed. Initially, a verification case is performed for both cases with available data showing the capability of the model to describe real data. Afterward, different scenarios are of concern investigating governmental actions and hospital infrastructure, evaluating their evolution until the end of 2020. Numerical simulations clearly show that social isolation, guided by governmental and individual actions, is essential to reduce the infected populations and the total period of the crisis. Results based on actual data show that the number of deaths can vary from 40 thousand to 2.5 million depending on the social isolation level and the hospital infrastructure. In addition, early governmental actions are essential to ensure smaller population infectious and the absence of shortage of hospital infrastructure for the part of the infectious population requiring assistance, for which numerical simulations indicate a total number of deaths below 200. Therefore, simulations present differences from 200 to 2.5 million deaths, showing that a central coordination is essential to save a huge number of lives. Different qualitative behaviors can be expected depending on social isolation levels. On the one hand, it is possible to observe a peak-vanish infectious curve representing a rapid dramatic crisis. On the other hand, COVID-19 dynamics can present a plateau behavior, spreading the crisis for a long period of time, being even more dramatic in terms of the number of deaths. Subsequent waves are also possible, establishing even more dramatic scenarios. Although the mathematical model can be improved in order to include more phenomenological information that can increase its capability to describe different scenarios, 
it should be pointed out that numerical simulations seem to be coherent with available data, being an important tool that can be useful for public health planning.

Acknowledgements The authors would like to acknowledge the support of the Brazilian Research Agencies CNPq, CAPES and FAPERJ.

\section{Declarations}

Conflict of interest The authors declare that they have no conflict of interest.

\section{References}

1. Brazilian Ministry of Health. CNESNet-Cadastro Nacional de Estabelecimentos de Saúde". http://cnes2.datasus. gov.br/Mod_Ind_Tipo_Leito.asp?VEstado=00. Accessed 20 May 2020 (2020).

2. Brazilian Ministry of Health. DATASUS-CNES-Recursos Físicos". http://tabnet.datasus.gov.br/cgi/deftohtm. exe?cnes/cnv/leiutibr.def. Accessed 20 May 2020 (2020)

3. Breto, C., He, D., Ionides, E.L., King, A.A.: Time series analysis via mechanistic models. Ann. Appl. Stat. 3(1), 319-348 (2009). https://doi.org/10.1214/08-AOAS201

4. Canabarro, A., Tenório, E., Martins, R., Martins, L., Brito, S., Chaves, R.: Data-driven study of the COVID-19 pandemic via age-structured modelling and prediction of the health system failure in Brazil amid diverse intervention strategies. medRxiv (preprint-15 April 2020). https://doi. org/10.1101/2020.04.03.20052498 (2020)

5. Car, Z., Šegota, S.B., Anđelić, N., Lorencin, I., Mrzljak, V.: Modeling the spread of COVID-19 infection using a multilayer perceptron. Comput. Math. Methods Med. 2020, Article ID 571471 (2020)

6. Castro, M.C., de Carvalho, L.R., Chin, T., Kahn, R., Franca, G.V.A., Macario, E.M., de Oliveira, W.K.: Demand for hospitalization services for COVID-19 patients in Brazil medRxiv (preprint-April 1, 2020). https://doi.org/10. 1101/2020.03.30.20047662 (2020)

7. Castro, M., Ares, S., Cuesta, J.A., Manrubi, S.: The turning point and end of an expanding epidemic cannot be precisely forecast. Proc. Natl. Acad. Sci. USA 117(42), Article 26191

8. Chen, T.-M., Rui, J., Wang, W.-P., Zhao, Z.-Y., Cui, J.-A., Yin, L.: A mathematical model for simulating the phasebased transmissibility of a novel coronavirus. Infect. Dis. Poverty (2020). https://doi.org/10.1186/s40249-020-006403

9. Ferguson, N.M., Laydon, D., Nedjati-Gilani, G., et al.: Impact of Non-Pharmaceutical Interventions (NPIs) to Reduce COVID-19 Mortality and Healthcare Demand. Imperial College, London (2020). https://doi.org/10.25561/ 77482

10. He, D., Dushoff, J., Day, T., Ma, J., Earn, D.J.D.: Inferring the causes of the three waves of the 1918 influenza pandemic in England and Wales. Proc R Soc B (2013). https://doi.org/10.1098/rspb.2013.1345

11. He, D., Ionides, E.L., King, A.A.: Plug-and-play inference for disease dynamics: measles in large and small populations as a case study. J. R. Soc. Interface 7, 271-283 (2010). https://doi.org/10.1098/rsif.2009.0151

12. Kuhl, E.: Data-driven modeling of COVID-19-Lessons learned. Extreme Mech. Lett. 40, Article 100921 (2020)

13. Li, R., Pei, S., Chen, B., Song, Y., Zhang, T., Yang, W., Shaman, J.: Substantial undocumented infection facilitates the rapid dissemination of novel coronavirus (SARSCoV2). Science (2020). https://doi.org/10.1126/science. abb3221

14. Li, Q., Med, M., Guan, X., Wu, P., Wang, X., Zhou, L., et al.: Early transmission dynamics in Wuhan, China, of novel coronavirus-infected pneumonia. N. Engl. J. Med. (2020). https://doi.org/10.1056/NEJMoa2001316

15. Lin, Q., Zhao, S., Gao, D., Lou, Y., Yang, S., Musa, S.S., Wang, M.H., Cai, Y., Wang, W., Yang, L., Hee, D.: A conceptual model for the coronavirus disease 2019 (COVID-19) outbreak in Wuhan, China with individual reaction and governmental action. Int. J. Infect. Dis. 93, 211-216 (2020)

16. López, L., Rodó, X.: A modified SEIR model to predict the COVID-19 outbreak in Spain and Italy: simulating control scenarios and multi-scale epidemics. medRxiv (preprintMarch 26, 2020). https://doi.org/10.1101/2020.03.27. 20045005

17. Lyra, W., do Nascimento Jr, J.D., Belkhiria, J., de Almeida, L., Chrispim, P.P.M., de Andrade, I.: COVID-19 pandemics modeling with SEIR $(+\mathrm{CAQH})$, social distancing, and age stratification. The effect of vertical confinement and release in Brazil. medRxiv (preprint-April 21, 2020) (2020). https://doi.org/10.1101/2020.04.09.20060053.

18. Prem, K., Liu, Y., Russell, T.W., Kucharski, A.J., Eggo, R.M., Davies, N.: The effect of control strategies to reduce social mixing on outcomes of the COVID-19 epidemic in Wuhan, China: a modelling study. Lancet Public Health (2020). https://doi.org/10.1016/S2468-2667(20)30073-6

19. Rihan, F.A., Al-Salti, N.S., Anwar, M.-N.Y.: Dynamics of coronavirus infection in human. AIP Conf. Proc. (2018). https://doi.org/10.1063/1.5045415

20. Riou, J., Althaus, C.L.: Pattern of early human-to-human transmission of Wuhan 2019 novel coronavirus (2019nCoV), December 2019 to January 2020. Euro Surveill (2020). https://doi.org/10.2807/1560-7917.ES.2020.25.4. 2000058

21. Savi, P.V., Savi M.A., Borges B.: A mathematical description of the dynamics of coronavirus disease 2019 (COVID-19): a case study of Brazil. Comput. Mathe. Methods Med. 2020, Article 9017157 (2020)

22. Savi, M.A.: Chaos and order in biomedical rhythms. J. Braz. Soc. Mech. Sci. Eng. 27(2), 157-169 (2005)

23. Worldometer, "COVID-19 Coronavirus Pandemic". https://www.worldometers.info/coronavirus/ (2020). Accessed 20 May 2020.

24. Weissman, G.E., Crane-Droesch, A., Chivers, C., et al.: Locally informed simulation to predict hospital capacity needs during the COVID-19 pandemic. Ann. Intern. Med. (2020). https://doi.org/10.7326/M20-1260 
25. Wu, J.T., Leung, K., Leung, G.M.: Nowcasting and forecasting the potential domestic and international spread of the 2019-nCoV outbreak originating in Wuhan, China: a modelling study. Lancet 395, 689-697 (2020). https://doi. org/10.1016/S0140-6736(20)30260-9

26. Zuo, M., Khosa, S.K., Ahmad, Z., Almaspoor, Z.: Comparison of COVID-19 pandemic dynamics in Asian countries with statistical modeling. Comput. Math. Methods Med. 2020, Article ID 4296806 (2020)

Publisher's Note Springer Nature remains neutral with regard to jurisdictional claims in published maps and institutional affiliations. 